\title{
Generalized Exponential Distribution
}

\section{Estimation of parameters using modifications in methods of Ranked Set Sampling}

\author{
Vyomesh Nandurbarkar · Ashok \\ Shanubhogue
}

Received: date / Accepted: date

\begin{abstract}
In this study, we estimate the parameters of the Generalized Exponential Distribution using Moving Extreme Ranked Set Sampling (MERSS). Using the maximum likelihood estimation method, we derive the expressions. MERSS estimates are compared with estimates obtained by simple random sampling (SRS) using a real data set. We also study the other variations of the methods of Ranked Set Sampling like Quartile Ranked Set Sampling(QRSS), Median Ranked Set Sampling(MRSS) and Flexible Ranked Set Sampling(FLERSS) (a scheme based on QRSS and MRSS). For known shape parameter values, we present coefficients for linear combinations of order statistics for least squares estimates. Here, the expressions are derived through maximum likelihood, and the estimates are calculated numerically. Simulated results indicate that estimates generated using least-squares and the maximum likelihood method for Ranked Set Sampling (RSS) perform better than those generated using Simple Random Sampling (SRS). Asymptotically, MERSS outperforms SRS, QRSS, MRSS, and FLERSS.
\end{abstract}

Keywords ranked set sampling · moving extremes ranked set sampling · maximum likelihood estimator · Fisher information number · Mean Square Error

Mathematics Subject Classification (2020) 62F07 62 F10 62 D05

\footnotetext{
Vyomesh Nandurbarkar

Department of Statistics, Sardar Patel University, Vallabh Vidyanagar, 388120

Gujarat,India Tel.: +91-9428696968

E-mail: vyomesh230404@gmail.com

Present address: Department of Mathematical Sciences, Charotar University of Science and

Technology, Changa 388 421, Anand, Gujarat, India
} 


\section{Introduction}

Consider a continuous random variable $X$ follows three parameter Generalized Exponential distribution $(\mathrm{GE}(\alpha, \mu, \sigma))$

$$
G(x ; \alpha, \mu, \sigma)=\left(1-e^{-(x-\mu) / \sigma}\right)^{\alpha}, \quad \alpha, \sigma, x>\mu>0
$$

The probability density function is

$$
g(x ; \alpha, \mu, \sigma)=\frac{\alpha}{\sigma}\left(1-e^{-(x-\mu) / \sigma}\right)^{\alpha-1} e^{-(x-\mu) / \sigma} \quad \alpha, \sigma, x>\mu>0
$$

Where $\alpha$ is the shape parameter, $\mu$ is the location parameter and $\sigma$ is the scale parameter. Substituting $\mu=0$ and $\beta=1 / \sigma$ in (1) and (2) we have

$$
\begin{gathered}
G(x ; \alpha, \beta)=\left(1-e^{-\beta x}\right)^{\alpha}, \quad \alpha, \beta, x>0 \\
g(x ; \alpha, \beta)=\alpha \beta\left(1-e^{-\beta x}\right)^{\alpha-1} e^{-\beta x} \quad \alpha, \beta, x>0
\end{gathered}
$$

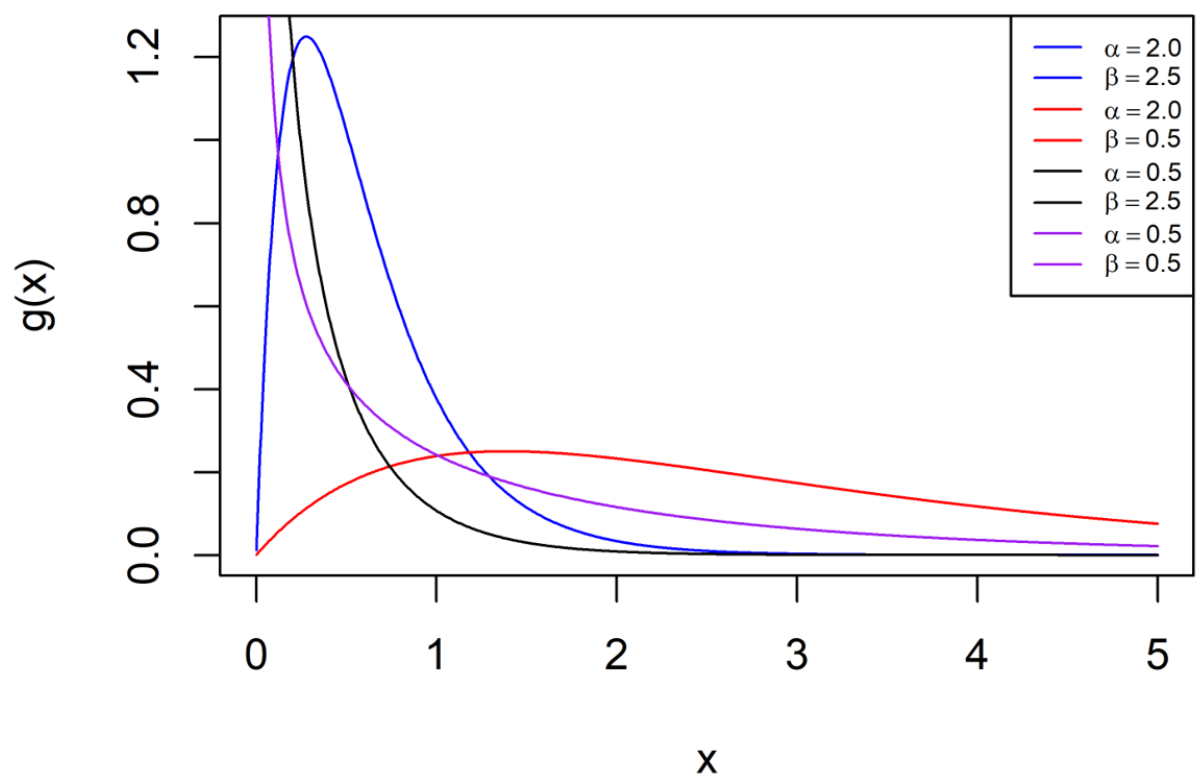

Fig. 1: $\alpha$ shape parameter, $\beta$ scale parameter, $\mu=0$ location parameter

As seen in (3), the distribution is of the type $[F(\cdot)]^{[/ a l p h a]}$ where $F(\cdot)$ is an absolutely continuous probability distribution function. The distribution (3) is introduced and studied in detail by Gupta and Kundu (1999). According to (Gupta and Kundu (2001)) when fitting positive life time data, the generalized 
exponential distribution is used as an alternative to the two parameter Weibull distribution and two parameter gamma distribution. Mean and variance of the distribution with density function given in (4) are

$$
\begin{aligned}
& E(X)=\frac{1}{\beta}(\psi(\alpha+1)-\psi(1)) \\
& V(X)=-\frac{1}{\beta^{2}}\left(\psi^{\prime}(\alpha+1)-\psi^{\prime}(1)\right)
\end{aligned}
$$

Where $\psi(\cdot)$ is the digamma function and $\psi^{\prime}(\cdot)$ is the derivative of $\psi(\cdot)$. The skewness and kurtosis both are independent of the scale parameter and they are decreasing function of the shape parameter $\alpha$.(Gupta and Kundu (2007))

The generalized exponential has increasing or decreasing hazard rate based on shape parameter $\alpha$. If $\alpha=1$, the distribution in (1) is two parameter exponetial distribution function. The survival function and hazard function are given by

$$
\begin{aligned}
& S(x ; \alpha, \mu, \sigma)=1-\left(1-e^{(x-\mu) / \sigma}\right)^{\alpha}, \quad x>\mu \\
& h(x ; \alpha, \mu, \sigma)=\frac{g(x ; \alpha, \mu, \sigma)}{S(x ; \alpha, \mu, \sigma)}=\frac{\alpha}{\sigma} \frac{\left(1-e^{-(x-\mu) / \sigma}\right)^{\alpha-1} e^{-(x-\mu) / \sigma}}{1-\left(1-e^{(x-\mu) / \sigma}\right)^{\alpha}}, \quad x>\mu
\end{aligned}
$$

Khan (1987) proposed the generalized exponential distribution and showed it to be a good fit to the survival data of black-headed gulls.

In reliability study, the system reliability is defined by a probability $R=$ $P(Y<X)$, where random variable $X$ refers to the strength of a component and random variable $Y$ refers to the stress on that component. Additionally, Kundu and Gupta (2005) examined the estimation of $R$ when $X$ and $Y$ are independent and distributed as generalized exponential distributions with common scale parameter and different shape parameters. Raqab et al. (2008) found the same solution using a three-parameter generalized exponential distribution. Using modified signed log-likelihood ratio statistics, Hajebi et al. (2012) have studied reliability intervals for $R$.

Chen and Lio (2010) studied the estimation of parameters of a generalized exponential distribution under progressive type-I interval censoring and derived estimates from real patient data.

Kundu and Gupta (2011) have discussed bivariate generalized exponential distributed whose marginal distributions are generalized exponential distributions and show it is a better fit than a bivariate exponential distribution.

Raqab Mohammad and Ahsanullah (2001) investigated the estimation of location and scale parameters using order statistics for a generalized exponential distribution (1). 


\section{Ranked Set Sampling}

Ranked set sampling (RSS) is a method that is cost-effective when the measurement of the sampling unit is expensive but would facilitate ranking. (see McIntyre (1952),Zehua Chen et al. (2004))

A ranked set sample is obtained by randomly selecting $m^{2}$ units from an infinite population. This units are then partitioned randomly into $m$ equal samples of $m$ units each. The units in the sample are ranked by judgement or visual inspection or by cheap (law cost) way, or by using auxiliary variables but without actual measurements. The unit with lowest rank is measured with respect to variable $X$ of interest and remaining units are discarded; a second sample of $m$ units are ranked without actual measurements. As a result, the second lowest unit in this set is measured with respect to variable $X$ of interest, while the rest are discarded. Once the largest unit in the last sample of size $m$ has been measured, the entire procedure is repeated $h$ times, yielding $n=m h$ measured units from $m^{2} h$ selected units. Compared to simple random sample, this ranked set sample also represents the entire population and is spread throughout the population. As the case in general with the RSS method, where the sampling units are selected without replacement, the estimation of population mean using RSS, and the relative precision of RSS estimate by comparison with SRS estimate, are discussed in Patil et al. (1995).

In Tahmasebi and Jafari (2014) the authors discuss estimation of the mean of a bivariate generalized exponential distribution using ranked set sampling.

Samuh and Qtait (2015) has examined the estimation of parameters in an Exponentiated exponential distribution based on Median ranked set sampling(MRSS) and found that MRSS provides more efficient estimates than SRS.

There are different modifications of the methods of RSS avialble in literature. Al-Odat and Al-Saleh (2001) introduced the concept of varied size RSS, which is known as Moving Extreme Ranked Set Sampling.

Eftekharian and Razmkhah (2016) discussed the unified ranked set sampling scheme to estimate the population mean and Eftekharian et al. (2021) discussed the flexible ranked set sampling scheme to estimate scale parameters of the exponential and normal distributions.

Our discussion assumes the sampling units are ranked without error for the characteristic of interest.In section 5 we discuss the estimation of shape and scale parameters of distribution given in (3) using MERSS method. In section 6 we discuss the unified ranked set sampling method to estimate shape and scale parameters of distribution given in (3). We consider the estimation of shape, location and scale parameters using the RSS method in section 7 . The steps for numerical computations are presented in section 9. Section 10 presents our findings. 


\section{Quartile Ranked Set Sampling}

According to Muttlak (2003), we note the Quartile Ranked Set Sampling as: If the sample size is even,

Step 1. Select for measurement from the first $m / 2$ samples the $\left[q_{1}(m+\right.$ 1)] th (the nearest integer) smallest rank. Where $q_{1}=0.25$

Step 2. Select for measurement from the second $m / 2$ samples the $\left(q_{u}(m+\right.$ 1)) th (the nearest integer) smallest rank. Where $q_{u}=0.75$

If the sample size is odd,

Step 1. Select for measurement from the first $(m-1) / 2$ samples the $\left[q_{1}(m+1)\right]$ th smallest rank. Where $q_{1}=0.25$

Step 2. Select for measurement from the second $(m-1) / 2$ samples the $\left[q_{u}(m+1)\right]$ th smallest rank. Where $q_{u}=0.75$

Step 3. From one sample the median for that sample for actual measurement.

\section{Median Ranked Set Sampling}

As mentioned by Muttlak (1997) we consider,

Step 1. If the sample size $m$ is odd, from each sample select for measurement the $((m+1) / 2)$ th smallest rank (the median of the sample)

Step 2. If the sample size $m$ is even, select for measurement from the first $m / 2$ samples the $(m / 2)$ th smallest rank and from the second $m / 2$ samples the $((m+2) / 2)$ th smallest rank.

\section{Moving Extreme Ranked Set Sampling}

Al-Odat and Al-Saleh (2001) has discussed moving extreme ranked set (MERSS) sampling to reduce the error of ranking . The procedure of MERSS is

Step 1. Select $m$ simple random samples of size $1,2,3, \ldots, m$ respectively.

Step 2. Order the sampling units of each of the samples by eye or by some other relatively inexpensive method, without actual measurement.

Step 3. Measure accurately the maximum order observation from the first set, the maximum ordered observation from the second set. The process continues in this way until the maximum ordered observation from the last $m$ th sample is measured.

Step 4. Repeat the Step 1. to Step 3. again, and measure the minimum ordered observation instead maximum ordered observation.

Step 5. The prcedure above is one cycle.The entire cycle can be repeated $h$ times to obtain a MERSS of size $n=2 m h$ 
Let $Y_{j i}, i=1,2, \ldots, m ; j=1,2, \ldots, h$ denotes a moving extreme ranked set sample with cumulative distribution function given in (3) and probability density function given in 2. Where $h$ is number of cyclels and $m$ is the set size.

$$
f_{Y_{j i}}(y)= \begin{cases}f_{X_{i(i: i)}^{j}}(y) & \text { Maximum } \\ f_{X_{i(1: i)}^{j}}(y) & \text { Minimum }\end{cases}
$$

Where $X_{i(i: i)}^{j}=\left\{X_{j 1(1: 1)}, \ldots, X_{j m(m: m)}\right\}$ and $X_{i(1: i)}^{j}=\left\{X_{j 1(1: 1)}, \ldots, X_{j m(1: m)}\right\}$

$$
\begin{aligned}
f_{X_{i(i: i)}^{j}(y)} & =i[G(y)]^{i-1} g(y) \\
& =i\left[\left(1-e^{-\beta y}\right)^{\alpha}\right]^{i-1} \alpha \beta\left(1-e^{-\beta y}\right)^{\alpha-1} e^{-\beta y} \\
& =\alpha \beta i\left(1-e^{-\beta y}\right)^{i \alpha-1} e^{-\beta y}
\end{aligned}
$$

and

$$
\begin{aligned}
f_{X_{i(1: i)}^{j}(y)} & =i[1-G(y)]^{i-1} g(y) \\
& =i\left[1-\left(1-e^{-\beta y}\right)^{\alpha}\right]^{i-1} \alpha \beta\left(1-e^{-\beta y}\right)^{\alpha-1} e^{-\beta y} \\
& =\alpha \beta i\left(1-e^{-\beta y}\right)^{\alpha-1}\left[1-\left(1-e^{-\beta y}\right)^{\alpha}\right]^{i-1} e^{-\beta y}
\end{aligned}
$$

\subsection{Likelihood Function}

Let $u_{j i}$ represents the observed values of $X_{i(i: i)}^{j}$ and $v_{j i}$ represents the observed values of $X_{i(1: i)}^{j}$, then

$$
\begin{aligned}
L_{M E R S S, \text { minimum }} & =\prod_{j=1}^{h} \prod_{i=1}^{m} f_{Y_{j i}}\left(v_{j i}\right) \\
& =\prod_{j=1}^{h} \prod_{i=1}^{m} \alpha \beta i\left(1-e^{-\beta v_{j i}}\right)^{\alpha-1}\left[1-\left(1-e^{-\beta v_{j i}}\right)^{\alpha}\right]^{i-1} e^{-\beta v_{j i}} \\
L_{M E R S S, \text { maximum }} & =\prod_{j=1}^{h} \prod_{i=1}^{m} f_{Y_{j i}}\left(u_{j i}\right) \\
& =\prod_{j=1}^{h} \prod_{i=1}^{m} \alpha \beta i\left(1-e^{-\beta u_{j i}}\right)^{i \alpha-1} e^{-\beta u_{j i}}
\end{aligned}
$$


Define, for $i=1,2, \ldots, m, \quad j=1,2, \ldots, h$

$$
\begin{gathered}
a_{j i}=\left\{\begin{array}{lll}
1, & \text { for } & \text { observed minimum } \\
0, & \text { for } & \text { observed maximum }
\end{array}\right. \\
b_{j i}=\left\{\begin{array}{lll}
0, & \text { for } & \text { observed minimum } \\
1, & \text { for } & \text { observed maximum }
\end{array}\right.
\end{gathered}
$$

Therefore,

$$
\begin{aligned}
& L_{M E R S S}=\prod_{j=1}^{h} \prod_{i=1}^{m}\left\{f_{Y_{j i}}\left(v_{j i}\right)\right\}^{a_{j i}}\left\{f_{Y_{j i}}\left(u_{j i}\right)\right\}^{b_{j i}} \\
& =\prod_{j=1}^{h} \prod_{i=1}^{m}\left[\alpha \beta i\left(1-e^{-\beta v_{j i}}\right)^{\alpha-1}\left\{1-\left(1-e^{-\beta v_{j i}}\right)^{\alpha}\right\}^{i-1} e^{-\beta v_{j i}}\right]^{a_{j i}} \\
& {\left[\alpha \beta i\left(1-e^{-\beta u_{j i}}\right)^{i \alpha-1} e^{-\beta u_{j i}}\right]^{b_{j i}}} \\
& \log L_{M E R S S}=\sum_{j=1}^{h} \sum_{i=1}^{m}\left[\log \left\{f_{Y_{j i}}\left(v_{j i}\right)\right\}^{a_{j i}}+\log \left\{f_{Y_{j i}}\left(u_{j i}\right)\right\}^{b_{j i}}\right] \\
& =\sum_{j=1}^{h} \sum_{i=1}^{m}\left[a_{j i} \log \left\{\alpha \beta i\left(1-e^{-\beta v_{j i}}\right)^{\alpha-1}\left\{1-\left(1-e^{-\beta v_{j i}}\right)^{\alpha}\right\}^{i-1} e^{-\beta v_{j i}}\right\}\right. \\
& \left.+b_{j i} \log \left\{\alpha \beta i\left(1-e^{-\beta u_{j i}}\right)^{i \alpha-1} e^{-\beta u_{j i}}\right\}\right] \\
& =C_{6}+\sum_{j=1}^{h} \sum_{i=1}^{m}\left[a_{j i}\left\{\log i+(\alpha-1) \log \left(1-e^{-\beta v_{j i}}\right)+(i-1) \log \left(1-\left(1-e^{-\beta v_{j i}}\right)^{\alpha}\right)-\beta v_{j i}\right\}\right. \\
& \left.+b_{j i}\left\{\log i+(i \alpha-1) \log \left(1-e^{-\beta u_{j i}}\right)-\beta u_{j i}\right\}\right]
\end{aligned}
$$

Where $C_{6}=2 m h\{\log \alpha+\log \beta\}$

\subsection{Estimates}

$$
\begin{aligned}
& \frac{\partial \log L_{M E R S S}}{\partial \alpha}=\frac{2 m h}{\alpha}+\sum_{j=1}^{h} \sum_{i=1}^{m} {\left[a_{j i} \log \left(1-e^{-\beta v_{j i}}\right)\left\{\frac{1-i\left(1-e^{-\beta v_{j i}}\right)^{\alpha}}{1-\left(1-e^{-\beta v_{j i}}\right)^{\alpha}}\right\}\right.} \\
&\left.+b_{j i}\left\{i \log \left(1-e^{-\beta u_{j i}}\right)\right\}\right]
\end{aligned}
$$


The mle of $\alpha$ which depends upon $\beta$ is the solution of equation $\frac{\partial \log L_{M E R S S}}{\partial \alpha}=0$

$$
\begin{aligned}
& \frac{\partial \log L_{M E R S S}}{\partial \beta}=\frac{2 m h}{\beta}+\sum_{j=1}^{h} \sum_{i=1}^{m} {\left[a_{j i} v_{j i}\left\{\frac{e^{-\beta v_{j i}}}{\left(1-e^{-\beta v_{j i}}\right)} \frac{(\alpha-1)-(i \alpha-1)\left(1-e^{-\beta v_{j i}}\right)^{\alpha}}{\left(1-\left(1-e^{-\beta v_{j i}}\right)^{\alpha}\right)}-1\right\}\right.} \\
&\left.-b_{j i} u_{j i}\left\{\frac{(i \alpha-1) e^{-\beta u_{j i}}}{\left(1-e^{-\beta u_{j i}}\right)}-1\right\}\right]
\end{aligned}
$$

The mle of $\beta$ which depends upon $\alpha$ is the solution of equation $\frac{\partial \log L_{M E R S S}}{\partial \beta}=0$

5.3 The Fisher Information (FI) matrix

We compute numerically the elements of Fisher Information Matrix

$$
I_{M E R S S}(\alpha, \beta)=-\frac{1}{2 m h}\left[\begin{array}{l}
E\left(\frac{\partial^{2} \log L}{\partial \alpha^{2}}\right) E\left(\frac{\partial^{2} \log L}{\partial \alpha \partial \beta}\right) \\
E\left(\frac{\partial^{2} \log L}{\partial \alpha \partial \beta}\right) E\left(\frac{\partial^{2} \log L}{\partial \beta^{2}}\right)
\end{array}\right]
$$

Where,

$$
\begin{aligned}
& \frac{\partial^{2} \log L_{M E R S S}}{\partial \alpha^{2}}=-\frac{m h}{\alpha^{2}}-\sum_{j=1}^{h} \sum_{i=1}^{m} a_{j i}\left[\frac{(i-1)\left(1-e^{\left.-\beta v_{j i}\right)^{\alpha} \log \left(1-e^{-\beta v_{j i}}\right)}\right.}{\left(1-\left(1-e^{-\beta v_{j i}}\right)\right)^{2}}\right] \\
& \frac{\partial^{2} \log L_{M E R S S}}{\partial \beta^{2}}=-\frac{m h}{\beta^{2}}+\sum_{j=1}^{h} \sum_{i=1}^{m}\left[a_{j i}\left\{\frac{e^{-\beta v_{j i} v_{j i}}}{\left(1-e^{-\beta v_{j i}}\right)}\right\}^{2}\right. \\
&\left\{\frac{(\alpha-1)-(\alpha i(i+1)-\alpha-1)\left(1-e^{-\beta v_{j i}}\right)^{\alpha}}{\left(1-\left(1-e^{-\beta v_{j i}}\right)^{\alpha}\right)^{2}}\right\} \\
&\left.-b_{j i}\left\{\frac{(i \alpha-1) e^{-\beta u_{j i}} u_{j i}^{2}}{\left(1-e^{-\beta u_{j i}}\right)^{2}}\right\}\right]
\end{aligned}
$$




$$
\begin{aligned}
\frac{\partial \log L_{M E R S S}}{\partial \alpha \partial \beta} & =\sum_{j=1}^{h} \sum_{i=1}^{m}\left[a_{j i}\left\{\frac{e^{-\beta v j i} v_{j i}}{1-e^{-\beta v_{j i}}}\right\}\right. \\
& \left\{\frac{1-i\left(1-e^{-\beta v_{j i}}\right)^{\alpha}}{1-\left(1-e^{-\beta v_{j i}}\right)^{\alpha}}-\frac{\alpha(i-1)\left(1-e^{-\beta v_{j i}}\right)^{\alpha} \log \left(1-e^{-\beta v_{j i}}\right)}{\left(1-\left(1-e^{-\beta v_{j i}}\right)^{\alpha}\right)^{2}}\right\} \\
& \left.-b_{j i}\left\{i \frac{e^{-\beta u j i} u_{j i}}{1-e^{-\beta u_{j i}}}\right\}\right]
\end{aligned}
$$

The components of Information Matrix (15) can also be obtained as

$$
I_{M E R S S}(\alpha, \beta)=I_{M E R S S, \text { maximum }}(\alpha, \beta)+I_{M E R S S, \text { minimum }}(\alpha, \beta)
$$

Denoting $G\left(\beta v_{j i}\right)=\left(1-e^{-\beta v_{j i}}\right)$ and $g\left(\beta v_{j i}\right)=e^{-\beta v_{j i}}$ the components of matrix $I_{M E R S S, \text { maximum }}(\alpha, \beta)$ are, $\left[\begin{array}{ll}E\left(a_{11}\right) & E\left(a_{12}\right) \\ E\left(a_{21}\right) & E\left(a_{22}\right)\end{array}\right]$ where

$$
\begin{aligned}
a_{11} & =-\frac{h m}{\alpha^{2}} \\
a_{21}=a_{12} & =\sum_{j=1}^{h} \sum_{i=1}^{m} i u_{j i} \frac{g\left(\beta u_{j i}\right)}{G\left(\beta u_{j i}\right)} \\
a_{22} & =-\frac{h m}{\beta^{2}}+\sum_{j=1}^{h} \sum_{i=1}^{m} u_{j i}^{2}\left[(i \alpha-1)\left\{\frac{G\left(\beta u_{j i}\right) g^{\prime}\left(\beta u_{j i}\right)-\left(g\left(\beta u_{j i}\right)\right)^{2}}{\left(G\left(\beta u_{j i}\right)\right)^{2}}\right\}\right]
\end{aligned}
$$

Therefore

$$
\begin{gathered}
E\left(a_{11}\right)=-\frac{h m}{\alpha^{2}} \\
E\left(a_{22}\right)=-\frac{-h m}{\beta^{2}}-\sum_{j=1}^{h} \sum_{i=1}^{m} \frac{i \alpha(i \alpha-1)}{(i \alpha-2)} \\
{\left[(\psi(i \alpha+1)+\gamma)^{2}-\psi^{\prime}(i \alpha+1)+\frac{\pi^{2}}{6}\right]} \\
E\left(a_{12}\right)=\sum_{j=1}^{h} \sum_{i=1}^{m} \frac{i}{i \alpha-1}(\psi(i \alpha+1)+\gamma), i \alpha \neq 1, i \alpha \neq 2
\end{gathered}
$$


Also the components of matrix $I_{M E R S S \text {,minimum }}(\alpha, \beta)$ are, $\left[\begin{array}{ll}E\left(b_{11}\right) & E\left(b_{12}\right) \\ E\left(b_{21}\right) & E\left(b_{22}\right)\end{array}\right]$ where

$$
\begin{gathered}
b_{11}=-\frac{h m}{\alpha^{2}}-\sum_{j=1}^{h} \sum_{i=1}^{m}(i-1)\left(\log G\left(\beta v_{j i}\right)\right)^{2} \frac{G\left(\beta v_{j i}\right)^{\alpha}}{1-G\left(\beta v_{j i}\right)^{\alpha}} \\
b_{21}=b_{12}=\sum_{j=1}^{h} \sum_{i=1}^{m} v_{j i}\left[\frac{\left(G\left(\beta v_{j i}\right)\right)^{\alpha-1} g\left(\beta v_{j i}\right)}{1-\left(G\left(\beta v_{j i}\right)\right)^{\alpha}}\right. \\
\left.\quad\left\{-(i-1)-\alpha(i-1) \frac{\log G\left(\beta v_{j i}\right)}{1-\left(G\left(\beta v_{j i}\right)\right)^{\alpha}}\right\}+\frac{g\left(\beta v_{j i}\right)}{G\left(\beta v_{j i}\right)}\right] \\
b_{22}=-\frac{h m}{\beta^{2}}+\sum_{j=1}^{h} \sum_{i=1}^{m} v_{j i}^{2}\left[\left\{(\alpha-1)-(i-1) \alpha \frac{G\left(\beta v_{j i}\right)^{\alpha}}{1-\left(G\left(\beta v_{j i}\right)\right)^{\alpha}}\right\}\right. \\
\left\{\frac{G\left(\beta v_{j i}\right) g^{\prime}\left(\beta v_{j i}\right)-\left(g\left(\beta v_{j i}\right)\right)^{2}}{\left(G\left(\beta v_{j i}\right)\right)^{2}}\right\} \\
\left.-(i-1) \alpha \frac{\left(G\left(\beta v_{j i}\right)\right)^{\alpha-2}\left(g\left(\beta v_{j i}\right)\right)^{2}}{\left(1-\left(G\left(\beta v_{j i}\right)\right)^{\alpha}\right)^{2}}\right]
\end{gathered}
$$

\subsection{Data Set}

In this section we demonstrate the performance of MERSS from the data (Table 1) used by Lieblein and Zelen (1956) and Gupta and Kundu (2001, 1999) representing the number of revolutions in millions before failure for each of the 23 ball bearings in the life test. When $m=2$, from the steps for MERSS we require $m(m+1)=6$ obervations, and we use number of cycles $h=3$. Therefore sample size of MERSS is $2 m h=12$. A simple random sample of the same size is selected without replacement from the data.

Table 1

Data set by Lieblein and Zelen (1956) and Gupta and Kundu (2001, 1999)

\section{Table 2}

The Estimates and Asymptotic efficiency using MERSS compared to SRS. The sample size $n=2 m h$, set size $m=2$ and number of cycles $h=3$.

$\begin{array}{rrrrr}17.88 & 28.92 & 33.00 & 41.52 & 42.12 \\ 45.60 & 48.40 & 51.84 & 51.96 & 54.12 \\ 55.56 & 67.80 & 68.64 & 68.64 & 68.88 \\ 84.12 & 93.12 & 98.64 & 105.12 & 105.84 \\ 127.92 & 128.04 & 173.40 & & \end{array}$

\begin{tabular}{lccl} 
Estimates & SRS & MERSS & AEFF \\
\hline$\hat{\alpha}$ & 7.06423 & 6.65388 & 1.073420 \\
$\hat{\beta}$ & 0.03508 & 0.03440 & 1.072517
\end{tabular}


Table 3

SRS: $95 \%$ CI

MERSS: 95\% CI

Calculated con-

fidence limits,

Fisher information

and Variance-

Covariance matrix

\begin{tabular}{llrll}
$\alpha$ & 0 & 15.55126 & 0 & 14.2623972 \\
$\beta$ & 0.01623 & 0.05392 & 0.0166774 & 0.0521169 \\
\hline \multicolumn{3}{c}{ SRS:FI } & \multicolumn{3}{c}{ MERSS:FI }
\end{tabular}

\begin{tabular}{|c|c|c|c|c|}
\hline & $\hat{\alpha}$ & $\hat{\beta}$ & $\hat{\alpha}$ & $\hat{\beta}$ \\
\hline$\hat{\alpha}$ & 0.43572 & -107.84840 & 0.5329473 & -136.844 \\
\hline \multirow[t]{3}{*}{$\hat{\beta}$} & -107.84841 & 43501.52840 & -136.844047 & 53163.388 \\
\hline & \multicolumn{2}{|c|}{ SRS: VC } & \multicolumn{2}{|c|}{ MERSS: VC } \\
\hline & $\hat{\alpha}$ & $\mathrm{b}$ & $\hat{\alpha}$ & $\hat{\beta}$ \\
\hline$\hat{\alpha}$ & 5.94012 & 0.01473 & 5.533824 & 0.0142442 \\
\hline$\hat{\beta}$ & 0.01473 & 0.0000595 & 0.014244 & 0.0000555 \\
\hline
\end{tabular}

\section{Unified (flexible) Ranked Set Sampling}

A Unified (flexible) RSS (FLERSS) with $M=h_{1}+h_{2}$ cycles is considered which containes $h_{1}$ cycles of Quartile Ranked Set Sampling (QRSS) and $h_{2}$ cycles of Median Ranked Set Sampling (MRSS). We consider in this section $h_{1}=h_{2}=h$.

Let $Y_{j i}, i=1,2, \ldots, m ; j=1,2, \ldots, h$ denotes a Quartile ranked set sample with cumulative distribution function given in (3) and probability density function given in (4). Where $h$ is number of cyclels and $m$ is the set size.

$$
f_{Y_{j i}}(y)= \begin{cases}f_{X_{q_{1}(m+1)}}(y) & \text { for } \quad i=1,2, \ldots \frac{m-1}{2} \\ f_{X_{q_{u}(m+1)}(y)}(y) & \text { for } \quad i=\frac{m-1}{2}+1, \frac{m-1}{2}+2, \ldots m-1 \\ f_{X_{0.5(m+1)}} & \text { for } \quad i=m\end{cases}
$$


$q_{1}=0.25, q_{u}=0.75$

Then,

$$
\begin{aligned}
f_{X_{q_{1}(m+1)}(y)=} C_{3}\left[\left\{1-e^{-\beta y}\right\}^{\alpha}\right]^{q_{1}(m+1)-1}\left[1-\left\{1-e^{-\beta y}\right\}^{\alpha}\right]^{m-q_{1}(m+1)} & \\
& \alpha \beta\left[1-e^{-\beta y}\right]^{\alpha-1} e^{-\beta y} \\
= & C_{3} \alpha \beta e^{-\beta y}\left[1-e^{-\beta y}\right]^{\alpha\left(q_{1}(m+1)\right)-1} \\
& {\left[1-\left\{1-e^{-\beta y}\right\}^{\alpha}\right]^{m-q_{1}(m+1)} } \\
f_{X_{\left(q_{u}(m+1)\right)}(y)=} C_{4}\left[\left\{1-e^{-\beta y}\right\}^{\alpha}\right]^{\left(q_{u}(m+1)\right)-1}\left[1-\left\{1-e^{-\beta y}\right\}^{\alpha}\right]^{m-\left(q_{u}(m+1)\right)} & \\
& \alpha \beta\left[1-e^{-\beta y}\right]^{\alpha-1} e^{-\beta y} \\
= & C_{4} \alpha \beta e^{-\beta y}\left[1-e^{-\beta y}\right]^{\alpha\left(q_{u}(m+1)\right)-1} \\
& {\left[1-\left\{1-e^{-\beta y}\right\}^{\alpha}\right]^{m-\left(q_{u}(m+1)\right)} }
\end{aligned}
$$

$$
\begin{gathered}
f_{X_{(0.5(m+1))}(y)=} C_{5}\left[\left\{1-e^{-\beta y}\right\}^{\alpha}\right]^{(0.5(m+1))-1}\left[1-\left\{1-e^{-\beta y}\right\}^{\alpha}\right]^{m-(0.5(m+1))} \\
\alpha \beta\left[1-e^{-\beta y}\right]^{\alpha-1} e^{-\beta y} \\
=C_{5} \alpha \beta e^{-\beta y}\left[1-e^{-\beta y}\right]^{\alpha(0.5(m+1))-1} \\
{\left[1-\left\{1-e^{-\beta y}\right\}^{\alpha}\right]^{m-(0.5(m+1))}}
\end{gathered}
$$

Let $Y_{j i}, i=1,2, \ldots, m ; j=1,2, \ldots, h$ denotes a Median ranked set sample with cumulative distribution function given in (3) and probability density function given in (4). Where $h$ is number of cyclels and $m$ is the set size. To derive the expressions for maximum likelihood equations, we consider general expressions using pdf of $r^{t h}$ order statistics. The PDf of $r$ th order statistics is

$$
\begin{aligned}
& f_{X_{(r)}}(y)=\frac{1}{B(r, m-r+1)} F^{r-1}(y)(1-F(y))^{m-r} f(y) \\
&= \frac{1}{B(r, m-r+1)}\left(\left(1-e^{-\beta y}\right)^{\alpha}\right)^{r-1}\left(1-\left(1-e^{-\beta y}\right)^{\alpha}\right)^{m-r} \\
& \alpha \beta\left(1-e^{-\beta y}\right)^{\alpha-1} e^{-\beta y}, \alpha>0, \beta>0
\end{aligned}
$$


The Likelihood function

$$
\begin{aligned}
& L=\prod_{j=1}^{h} \prod_{i=1}^{m} f_{Y_{(r)}}\left(y_{j i}\right) \\
&=\prod_{j=1}^{h} \prod_{i=1}^{m} \frac{1}{B(r, m-r+1)}\left(\left(1-e^{-\beta y_{j i}}\right)^{\alpha}\right)^{r-1} \\
&\left(\frac{\left.1-\left(1-e^{-\beta y_{j i}}\right)^{\alpha}\right)^{m-r} \alpha \beta\left(1-e^{\left.-\beta y_{j i}\right)^{\alpha-1}} e^{-\beta y_{j i}}\right.}{B(r, m-r+1)}\right)^{m h} \prod_{j=1}^{h} \prod_{i=1}^{m}\left(\left(1-e^{\left.-\beta y_{j i}\right)^{\alpha}}\right)^{r \alpha-1}\right. \\
&\left(1-\left(1-e^{-\beta y_{j i}}\right)^{\alpha}\right)^{m-r} \alpha \beta e^{-\beta y_{j i}} \\
& \log L=\sum_{j=1}^{h} \sum_{i=1}^{m} \log f_{Y_{(r)}}\left(y_{j i}\right) \\
&=C_{7}+m h(\log \alpha+\log \beta) \\
&+\sum_{j=1}^{h} \sum_{i=1}^{m}\left\{(r \alpha-1) \log \left(1-e^{-\beta y_{j i}}\right)\right. \\
&\left.+(m-r) \log \left(1-\left(1-e^{-\beta y_{j i}}\right)^{\alpha}\right)-\beta y_{j i}\right\}
\end{aligned}
$$

Where $C_{7}=\log \left(\frac{1}{B(r, m-r+1)}\right)$

6.1 The Likelihood Functions

$$
\frac{\partial \log L}{\partial \alpha}=\frac{2 m h}{\alpha}+\sum_{j=1}^{h} \sum_{i=1}^{m} \log \left(1-e^{-\beta y_{j i}}\right)\left\{\frac{r-m\left(1-e^{-\beta y_{j i}}\right)}{\left(1-\left(1-e^{-\beta y_{j i}}\right)^{\alpha}\right)}\right\}
$$

The mle of $\alpha$ which depends upon $\beta$ is the solution of equation $\frac{\partial \log L}{\partial \alpha}=0$

$$
\frac{\partial \log L}{\partial \beta}=\frac{2 m h}{\beta}+\sum_{j=1}^{h} \sum_{i=1}^{m} y_{j i}\left[\frac{e^{-\beta y_{j i}}}{\left(1-e^{-\beta y_{j i}}\right)}\left\{\frac{r-m\left(1-e^{-\beta y_{j i}}\right)^{\alpha}}{1-\left(1-e^{-\beta y_{j i}}\right)^{\alpha}}-1\right\}-1\right]
$$

The mle of $\beta$ which depends upon $\alpha$ is the solution of equation $\frac{\partial \log L}{\partial \beta}=0$ 


$$
\begin{aligned}
& \frac{\partial^{2} \log L}{\partial \alpha^{2}}=-\frac{2 m h}{\alpha^{2}}-\sum_{j=1}^{h} \sum_{i=1}^{m}\left\{\frac{(m-r)\left(1-e^{-\beta y_{j i}}\right)\left(\log \left(1-e^{-\beta y_{j i}}\right)\right)^{2}}{\left(1-\left(1-e^{-\beta y_{j i}}\right)^{\alpha}\right)^{2}}\right\} \\
& \frac{\partial^{2} \log L}{\partial \beta^{2}}=-\frac{2 m h}{\beta^{2}}-\sum_{j=1}^{h} \sum_{i=1}^{m} y_{j i}^{2} \frac{e^{-\beta y_{j i}}}{\left(1-e^{-\beta y_{j i}}\right)^{2}}\left[\left\{\frac{r-m\left(1-e^{-\beta y_{j i}}\right)^{\alpha}}{1-\left(1-e^{-\beta y_{j i}}\right)^{\alpha}}-1\right\}\right. \\
& \left.+e^{-\beta y_{j i}} \frac{\alpha(m+r)\left(1-e^{-\beta y_{j i}}\right)^{\alpha}}{\left(1-\left(1-e^{-\beta y_{j i}}\right)^{\alpha}\right)^{2}}\right] \\
& \frac{\partial \log L}{\partial \alpha \partial \beta}=\sum_{j=1}^{h} \sum_{i=1}^{m} \frac{y_{j i} e^{-\beta y_{j i}}}{\left(1-e^{-\beta y_{j i}}\right)}\left[\left\{\frac{r-m\left(1-e^{-\beta y_{j i}}\right)^{\alpha}}{\left(1-\left(1-e^{-\beta y_{j i}}\right)^{\alpha}\right)}\right\}\right. \\
& \left.-\frac{\alpha(m-r)\left(1-e^{-\beta y_{j i}}\right)^{\alpha} \log \left(1-e^{-\beta y_{j i}}\right)}{\left(1-\left(1-e^{-\beta y_{j i}}\right)^{\alpha}\right)^{2}}\right]
\end{aligned}
$$

6.2 Likelihhod function (Unified(Flexible) Ranked Set Sampling

Define, for $j=1,2, \ldots, h$

$$
\begin{aligned}
& a_{j i}=\left\{\begin{array}{lll}
1, & \text { for } & i=1,2, \ldots, k \\
0, & \text { for } & i=k+1, k+2, \ldots, m
\end{array}\right. \\
& b_{j i}=\left\{\begin{array}{lll}
0, & \text { for } & i=1,2, \ldots, k \\
1, & \text { for } & i=k+1, k+2, \ldots, m-1 \\
0, & i=m
\end{array}\right. \\
& c_{j i}=\left\{\begin{array}{lll}
0, & \text { for } & i=1,2, \ldots, m-1 \\
1, & \text { for } & i=m
\end{array}\right.
\end{aligned}
$$

Let $L_{F L E R S S}, L_{Q R S S}$ and $L_{M R S S}$ denote respectively the likelihood functions of the sampling scheme FLERSS, QRSS and MRSS. Then,

$$
L_{F L E R S S}=L_{Q R S S} L_{M R S S}
$$

Where

$$
\begin{aligned}
L_{Q R S S ; m \text { Odd }}(\alpha, \beta) & =\prod_{j=1}^{h} \prod_{i=1}^{k} f_{Y_{i}}\left(y_{j i}\right) \prod_{j=1}^{h} \prod_{i=k+1}^{m-1} f_{Y_{i}}\left(y_{j i}\right) \prod_{j=1}^{h} f_{Y_{m}}\left(y_{j m}\right) \\
& =\prod_{j=1}^{h} \prod_{i=1}^{m}\left[f_{Y_{i}}\left(y_{j i}\right)\right]^{a_{j i}}\left[f_{Y_{i}}\left(y_{j i}\right)\right]^{b_{j i}}\left[f_{Y_{i}}\left(y_{j i}\right)\right]^{c_{j i}}
\end{aligned}
$$


and

$$
L_{M R S S ; m \text { Odd }}(\alpha, \beta)=\prod_{j=1}^{h} \prod_{i=1}^{m} f_{Y_{i}}\left(y_{j i}\right)
$$

The Log-Likelihood function is

$$
\log L_{F L E R S S}=\log L_{Q R S S}+\log L_{M R S S}
$$

The normal equations are,

$$
\begin{aligned}
& \frac{\partial \log L_{F L E R S S}}{\partial \alpha}=\frac{\partial \log L_{Q R S S}}{\partial \alpha}+\frac{\partial \log L_{M R S S}}{\partial \alpha} \\
& \frac{\partial \log L_{F L E R S S}}{\partial \beta}=\frac{\partial \log L_{Q R S S}}{\partial \beta}+\frac{\partial \log L_{M R S S}}{\partial \beta}
\end{aligned}
$$

and the Fisher Information matrix is

$$
I_{F L E R S S}=I_{Q R S S}+I_{M R S S}
$$

By substituting $r=\left[q_{1}(m+1)\right], r=\left[q_{u}(m+1)\right]$ and $r=[0.5(m+1)]$ in $((27)$ to (31)) to compute the estimates by solving equations (36) to (39) numerically using $\mathrm{R}$ maxLik package (Henningsen and Toomet (2011)). Where $[x]$ represent nearest ingeter.

\subsection{Simulated Output}

In this section, we generate 5000 random numbers from the Generalized exponential distribution given in (3) when shape parameter $\alpha=0.5, \alpha=4.0$ and scale parameter $\beta=0.5$. The Tables (4) to (8) show the computatuions. The Authors have computed similar tables when $\alpha=4.0$ and $\beta=0.5$.

\section{Table 4}

Estimate of shape

\begin{tabular}{|c|c|c|c|c|c|c|}
\hline \multirow{5}{*}{$\begin{array}{l}\text { parameter } \\
(\alpha=0.5) \\
\text { for different } \\
\text { cles } h\end{array}$} & $h$ & MERSS & FLERSS & SRS & QRSS & MRSS \\
\hline & 2 & 0.53896 & 0.54630 & 0.54659 & 0.55437 & 0.55472 \\
\hline & 3 & 0.52533 & 0.54274 & 0.52727 & 0.54726 & 0.54693 \\
\hline & 4 & 0.52342 & 0.54262 & 0.52273 & 0.53064 & 0.53120 \\
\hline & 5 & 0.52049 & 0.52234 & 0.52378 & 0.52772 & 0.53414 \\
\hline
\end{tabular}
parameter

$(\alpha=0.5)$ for different cycles $h$

\section{$\hat{\alpha}$}


Table 5

Estimate of scale parameter $(\beta=0.5)$ for different cycles $h$

$\hat{\beta}$

\begin{tabular}{lrlrll}
\hline$h$ & MERSS & SRS & FLERSS & MRSS & QRSS \\
\hline 2 & 0.53098 & 0.54355 & 0.56170 & 0.56254 & 0.56320 \\
3 & 0.52868 & 0.52421 & 0.54429 & 0.55008 & 0.55307 \\
4 & 0.51752 & 0.52166 & 0.52656 & 0.53107 & 0.53135 \\
5 & 0.51453 & 0.51872 & 0.51908 & 0.53498 & 0.52426 \\
\hline
\end{tabular}

Table 6

Efficiency of MERSS for different cycles $h$.

\begin{tabular}{llll} 
Sampling & $h$ & $\hat{\alpha}$ & $\hat{\beta}$ \\
\hline SRS & 2 & 1.0136194 & 1.0578518
\end{tabular}
$\alpha=0.5, \beta=0.5$

\begin{tabular}{llll} 
& 3 & 1.0331492 & 1.0295447 \\
& 4 & 1.0366661 & 1.0174703 \\
QRSS & 5 & 1.0035649 & 1.0088355 \\
& 2 & 1.0292501 & 1.0594382 \\
& 3 & 1.0411325 & 1.0404958 \\
& 4 & 1.0148621 & 1.0261800 \\
MRSS & 5 & 1.0262226 & 1.0397384 \\
& 2 & 1.0285954 & 1.0606792 \\
& 3 & 1.0417569 & 1.0461465 \\
& 4 & 1.0137888 & 1.0267224 \\
FLERSS & 5 & 1.0138931 & 1.0189093 \\
& 2 & 1.0141577 & 1.0236766 \\
& 3 & 1.0036985 & 0.9915609 \\
& 4 & 0.9986808 & 1.0079921 \\
& 5 & 1.0063299 & 1.0081332 \\
\hline
\end{tabular}


Table 7

Asymptotic Efficiency of MERSS for different cycles $h$. $\alpha=0.5, \beta=0.5$

\begin{tabular}{llll}
\hline Sampling & Cycles & $\hat{\alpha}$ & $\hat{\beta}$ \\
\hline SRS & 2 & 6.54024 & 5.74138 \\
& 3 & 7.01180 & 5.67670 \\
& 4 & 7.09303 & 5.64129 \\
QRSS & 5 & 6.72755 & 5.64903 \\
& 2 & 2.93797 & 2.85718 \\
& 3 & 3.20964 & 2.92172 \\
MRSS & 4 & 3.04382 & 2.94519 \\
& 5 & 3.19900 & 3.09289 \\
& 3 & 4.96470 & 5.42898 \\
& 4 & 4.22723 & 5.27204 \\
& 5 & 4.96617 & 5.24216 \\
& 2 & 3.75883 & 3.88354 \\
& 3 & 3.80268 & 3.73140 \\
& 4 & 3.71812 & 3.83538 \\
& 5 & 3.80284 & 3.87356 \\
\hline
\end{tabular}


Table 8

$\alpha=0.5, \beta=0.5$ :

Observed Fisher Information (FI),Variance- Covariance(VC) and $95 \%$ confidence limits for different cycles $h$.

\begin{tabular}{|c|c|c|c|c|c|c|c|c|c|}
\hline \multirow[t]{2}{*}{ Sampling } & \multirow[t]{2}{*}{$h$} & \multirow{2}{*}{$\begin{array}{l}\text { Est } \\
\hat{\alpha}\end{array}$} & \multicolumn{2}{|c|}{ FI } & \multicolumn{2}{|c|}{$\mathrm{VC}$} & \multicolumn{2}{|c|}{$95 \%$ CI $(\alpha, \beta)$} & \multirow{2}{*}{$\begin{array}{l}\text { Width } \\
0.48581\end{array}$} \\
\hline & & & 109.33888 & -42.15818 & 0.01228 & 0.00812 & 0.30339 & 0.78920 & \\
\hline \multirow{7}{*}{ SRS } & & $\hat{\beta}$ & -42.15818 & 63.74626 & 0.00812 & 0.02106 & 0.23483 & 0.88857 & 0.65374 \\
\hline & 3 & $\hat{\alpha}$ & 158.35636 & -62.75473 & 0.00862 & 0.00583 & 0.34632 & 0.73916 & 0.39284 \\
\hline & & $\hat{\beta}$ & -62.75473 & 92.87910 & 0.00583 & 0.01470 & 0.28507 & 0.80352 & 0.51844 \\
\hline & 4 & $\hat{\alpha}$ & 205.31954 & -84.68729 & 0.00672 & 0.00449 & 0.37272 & 0.71251 & 0.33980 \\
\hline & & $\hat{\beta}$ & -84.68729 & 126.82636 & 0.00449 & 0.01088 & 0.30942 & 0.74370 & 0.43428 \\
\hline & 5 & $\hat{\alpha}$ & 270.42170 & -107.83260 & 0.00509 & 0.00349 & 0.37693 & 0.66776 & 0.29084 \\
\hline & & $\hat{\beta}$ & -107.83260 & 157.34370 & 0.00349 & 0.00875 & 0.32535 & 0.71281 & 0.38746 \\
\hline \multirow{8}{*}{ QRSS } & 2 & $\hat{\alpha}$ & 377.70090 & -197.56690 & 0.00551 & 0.00548 & 0.38700 & 0.72244 & 0.33544 \\
\hline & & $\hat{\beta}$ & -197.56690 & 198.77450 & 0.00548 & 0.01048 & 0.33162 & 0.79347 & 0.46185 \\
\hline & 3 & $\hat{\alpha}$ & 546.98160 & -289.46490 & 0.00395 & 0.00400 & 0.41213 & 0.68173 & 0.26960 \\
\hline & & $\hat{\beta}$ & -289.46490 & 285.32510 & 0.00400 & 0.00757 & 0.36300 & 0.73716 & 0.37416 \\
\hline & 4 & $\hat{\alpha}$ & 755.36690 & -395.90440 & 0.00288 & 0.00298 & 0.41857 & 0.64384 & 0.22527 \\
\hline & & $\hat{\beta}$ & -395.90440 & 383.52160 & 0.00298 & 0.00568 & 0.37285 & 0.68929 & 0.31645 \\
\hline & 5 & $\hat{\alpha}$ & 917.76280 & -483.69680 & 0.00242 & 0.00252 & 0.43266 & 0.63561 & 0.20295 \\
\hline & & $\hat{\beta}$ & -483.69680 & 463.77260 & 0.00252 & 0.00479 & 0.39236 & 0.67761 & 0.28525 \\
\hline \multirow{8}{*}{ MRSS } & 2 & $\hat{\alpha}$ & 388.56400 & -226.16270 & 0.00932 & 0.01159 & 0.34210 & 0.76664 & 0.42454 \\
\hline & & $\hat{\beta}$ & -226.16270 & 181.86150 & 0.01159 & 0.01991 & 0.25678 & 0.86962 & 0.61285 \\
\hline & 3 & $\hat{\alpha}$ & 576.44870 & -337.98210 & 0.00643 & 0.00801 & 0.37722 & 0.71731 & 0.34009 \\
\hline & & $\hat{\beta}$ & -337.98210 & 271.39520 & 0.00801 & 0.01366 & 0.30588 & 0.80026 & 0.49438 \\
\hline & 4 & $\hat{\alpha}$ & 804.84740 & -466.72150 & 0.00464 & 0.00587 & 0.38902 & 0.67226 & 0.28324 \\
\hline & & $\hat{\beta}$ & -466.72150 & 369.48410 & 0.00587 & 0.01012 & 0.32293 & 0.73978 & 0.41685 \\
\hline & 5 & $\hat{\alpha}$ & 1004.03010 & -585.54920 & 0.00376 & 0.00473 & 0.40201 & 0.65343 & 0.25142 \\
\hline & & $\hat{\beta}$ & -585.54920 & 464.71140 & 0.00473 & 0.00812 & 0.33973 & 0.70879 & 0.36906 \\
\hline \multirow{8}{*}{ FLERSS } & 2 & $\hat{\alpha}$ & 381.84520 & -213.11500 & 0.00706 & 0.00795 & 0.36503 & 0.72815 & 0.36312 \\
\hline & & $\hat{\beta}$ & -213.11500 & 189.15390 & 0.00795 & 0.01424 & 0.28933 & 0.79778 & 0.50844 \\
\hline & 3 & $\hat{\alpha}$ & 587.64660 & -326.04790 & 0.00468 & 0.00536 & 0.38559 & 0.66895 & 0.28336 \\
\hline & & $\hat{\beta}$ & -326.04790 & 284.36930 & 0.00536 & 0.00967 & 0.32061 & 0.72782 & 0.40721 \\
\hline & 4 & $\hat{\alpha}$ & 787.69010 & -434.77300 & 0.00352 & 0.00408 & 0.40136 & 0.64411 & 0.24275 \\
\hline & & $\hat{\beta}$ & -434.77300 & 375.14250 & 0.00408 & 0.00740 & 0.34547 & 0.69784 & 0.35237 \\
\hline & 5 & $\hat{\alpha}$ & 973.95640 & -540.90420 & 0.00288 & 0.00333 & 0.41494 & 0.63263 & 0.21768 \\
\hline & & $\hat{\beta}$ & -540.90420 & 467.15640 & 0.00333 & 0.00600 & 0.36196 & 0.67548 & 0.31352 \\
\hline \multirow{8}{*}{ MERSS } & 2 & $\hat{\alpha}$ & 783.94270 & -317.46380 & 0.00188 & 0.00149 & 0.44810 & 0.62982 & 0.18172 \\
\hline & & $\hat{\beta}$ & -317.46380 & 401.22460 & 0.00149 & 0.00367 & 0.40322 & 0.65875 & 0.25553 \\
\hline & 3 & $\hat{\alpha}$ & 1208.34490 & -476.24120 & 0.00123 & 0.00102 & 0.45317 & 0.59748 & 0.14431 \\
\hline & & $\hat{\beta}$ & -476.24120 & 573.77240 & 0.00102 & 0.00259 & 0.42362 & 0.63373 & 0.21012 \\
\hline & 4 & $\hat{\alpha}$ & 1584.19900 & -641.62640 & 0.00095 & 0.00078 & 0.46128 & 0.58557 & 0.12429 \\
\hline & & $\hat{\beta}$ & -641.62640 & 778.27960 & 0.00078 & 0.00193 & 0.42830 & 0.60674 & 0.17844 \\
\hline & 5 & $\hat{\alpha}$ & 1988.80690 & -804.86960 & 0.00076 & 0.00063 & 0.46529 & 0.57569 & 0.11040 \\
\hline & & $\hat{\beta}$ & -804.86960 & 971.66730 & 0.00063 & 0.00155 & 0.43505 & 0.59401 & 0.15896 \\
\hline
\end{tabular}




\section{Best Linear Unbiased Estimates}

Consider the transformation $Y=\beta X$ in (4) the probability density function of $Y$ is

$$
f(y ; \alpha)=\alpha\left(1-e^{-y}\right)^{\alpha} e^{-y}, \quad y>0
$$

Let $Y_{1}, Y_{2}, \ldots, Y_{n}$ be a random sample from the distribution (40. Then pdf of $r^{\text {th }}$ order statistic $Y_{r: n},(r=1,2, \ldots, n)$ is

$$
f_{r: n}(y)=\frac{1}{B(r, n-r+1)} F^{r-1}(y)[1-F(y)]^{n-r} f(y)
$$

Where $B(p, q)=\frac{\Gamma(p) \Gamma(q)}{\Gamma(p+q)}$

$$
\begin{aligned}
f_{r: n}(y) & =\frac{1}{B(r, n-r+1)} \sum_{i=0}^{n-r}(-1)^{i}\left(\begin{array}{c}
n-r \\
i
\end{array}\right) \alpha\left[1-e^{-y}\right]^{(r+i) \alpha-1} e^{-y} \\
& =\frac{1}{B(r, n-r+1)} \sum_{i=0}^{n-r}(-1)^{i}\left(\begin{array}{c}
n-r \\
i
\end{array}\right) \alpha(r+i) \frac{\left[1-e^{-y}\right]^{(r+i) \alpha-1}}{r+i} e^{-y} \\
& =\frac{1}{B(r, n-r+1)} \sum_{i=0}^{n-r} \frac{(-1)^{i}\left(\begin{array}{c}
n-r \\
i
\end{array}\right)}{r+i} f\left(y ; \alpha_{(r+i)}\right)
\end{aligned}
$$

For $(r=1,2, \ldots, n)$ the moment genrating function is

$$
\begin{aligned}
M_{Y_{r: n}}(t) & =\int_{0}^{\infty} f_{r: n}(y) e^{t y} d y \\
& =\int_{0}^{\infty} \frac{1}{B(r, n-r+1)} \sum_{i=0}^{n-r} \frac{(-1)^{i}\left(\begin{array}{c}
n-r \\
i
\end{array}\right)}{r+i} f\left(y ; \alpha_{(r+i)}\right) e^{t y} d y \\
& =\frac{\alpha}{B(r, n-r+1)} \sum_{i=0}^{n-r}(-1)^{i}\left(\begin{array}{c}
n-r \\
i
\end{array}\right) \frac{\Gamma(\alpha(r+i)) \Gamma(1-t)}{\Gamma(\alpha(r+i)+1-t)}, \quad t<1
\end{aligned}
$$

Setting $\left.M_{Y_{r: n}}^{\prime}(t)\right|_{t=0}$ we get

$$
\begin{aligned}
E\left(Y_{r: n}\right) & =\frac{1}{B(r, n-r+1)} \sum_{i=0}^{n-r} \frac{(-1)^{i}\left(\begin{array}{c}
n-r \\
i
\end{array}\right)}{r+i}[-\psi(1)+\psi(\alpha(r+i)+1)] \\
& =\frac{1}{B(r, n-r+1)} \sum_{i=0}^{n-r} \frac{(-1)^{i}\left(\begin{array}{c}
n-r \\
i
\end{array}\right)}{r+i}[\gamma+\psi(\alpha(r+i)+1)]
\end{aligned}
$$

Setting $\left.M_{Y_{r: n}}^{\prime \prime}(t)\right|_{t=0}$ we get

$$
\begin{array}{r}
E\left\{Y_{(i: n)}^{2}\right\}=\frac{1}{B(i, n-i+1)} \sum_{j=0}^{n-i(-1)^{j}\left(\begin{array}{c}
n-i \\
j
\end{array}\right)} \frac{1}{j+i}\{((i+j) \alpha+1)+\gamma]^{2} \\
\left.-\psi^{\prime}((i+j) \alpha+1)+\pi^{2} / 6\right\}
\end{array}
$$


Raqab Mohammad and Ahsanullah (2001) have derived the product moment of $r^{\text {th }}$ and $s^{\text {th }}$ order statistics,

$$
\begin{array}{r}
E\left\{Y_{(r: n)} Y_{(s: n)}\right\}=C_{r, s: n} \alpha^{2} \sum_{k=1}^{\infty} \sum_{i=0}^{n-s} \sum_{j=0}^{s-r-1}(-1)^{i+j}\left(\begin{array}{c}
s-r-1 \\
j
\end{array}\right)\left(\begin{array}{c}
n-s \\
i
\end{array}\right) \\
\frac{[\psi((s+i) \alpha+k+1)+\gamma]}{k[(s+i) \alpha+k][(r+j) \alpha+k]}
\end{array}
$$

Where

$$
C_{r, s: n}=\frac{n !}{(r-1) !(s-r-1) !(n-s) !}
$$

7.1 Estimation of scale parameter using SRS

Let $X_{1}, X_{2}, \ldots, X_{n}$ be a simple random sample from the pdf given in (2) and $X_{(1: n)}, X_{(2: n)}, X_{(3: n)}, \ldots, X_{(n: n)}$ are the order statistics then

$$
E\left\{X_{(r: n)}\right\}=u_{r} \sigma, \quad V\left\{X_{(r: n)}\right\}=\sigma^{2} w_{r r}, \quad \operatorname{Cov}\left(X_{(r: n)}, X_{(s: n)}\right)=\sigma^{2} w_{r s}
$$

Define $\mathbf{u}=\left(u_{1}, u_{2}, \ldots, u_{n}\right)_{n \times 1}$ be the vector of $u_{r}$ $\mathbf{X}=\left(X_{(1)}, X_{(2)}, X_{(3)}, \ldots, X_{(n)}\right)_{n \times 1}, \mathbf{w}_{n \times n}$ is the variance covariance matrix that is symmetric positive definite. The equations (46) in matrix form is

$$
E(\mathbf{X})=\sigma \mathbf{u}, V(\mathbf{X})=\sigma^{2} \mathbf{w}
$$

if $\hat{\sigma}_{\text {BLUESRS }}$ denotes the BLUE of $\sigma$ based on SRS, then

$$
\hat{\sigma}_{\text {BLUESRS }}=\left(\mathbf{u}^{\prime} \boldsymbol{\Omega u}\right)^{-\mathbf{1}} \mathbf{u}^{\prime} \mathbf{\Omega X}
$$

where $\boldsymbol{\Omega}=\mathbf{w}^{-\mathbf{1}}, \Delta=\left|\mathbf{u}^{\prime} \boldsymbol{\Omega} \mathbf{u}\right|$ and

$$
V\left(\hat{\sigma}_{\text {BLUESRS }}\right)=\left(\mathbf{u}^{\prime} \mathbf{\Omega u}\right) \sigma^{2} / \boldsymbol{\Delta}
$$

\subsection{Estimation of Scale Parameter using RSS}

We assume that the both parameters $\alpha$ and $\mu$ are known.

Let $X_{(11)}, X_{(22)}, X_{(33)}, \ldots, X_{(n n)}$ be a ranked set sample (cycle 1) from the pdf given in (3), then

$$
\begin{aligned}
& E\left\{X_{(i i)}\right\}=c_{i: n} \sigma \\
& V\left\{X_{(i i)}\right\}=d_{i: n} \sigma^{2}
\end{aligned}
$$


where $c_{i: n}=E\left\{Y_{(i i)}\right\}, d_{i: n}=V\left\{Y_{(i i)}\right\}$ and the expressions for $E\left\{Y_{(i i)}\right\}$ and $E\left\{Y_{(i i)}^{2}\right\}$ are same as given in (43) and (45). If $\hat{\sigma}_{\text {RSS }}$ denote the least squares estimate of $\sigma$ based on RSS, then

$$
\hat{\sigma}_{\mathrm{RSS}}=\frac{1}{\sum_{i=1}^{n} c_{i: n}^{2} / d_{i: n}} \sum_{i=1}^{n} \frac{c_{i: n} X_{i i}}{d_{i: n}}
$$

The variance of $\hat{\sigma}_{\mathrm{RSS}}$ is given by

$$
V\left(\hat{\sigma}_{\text {RSS-BLUE }}\right)=\sigma^{2} \frac{1}{\sum_{i=1}^{n} c_{i: n}^{2} / d_{i: n}}
$$

The relative precision (RP) in estimating $\hat{\sigma}$ using RSS is

$$
\mathrm{RP}_{1}=\frac{V\left(\hat{\sigma}_{B L U E S R S}\right)}{V\left(\hat{\sigma}_{\mathrm{RSS}-\mathrm{BLUE}}\right)}
$$

Table 9

Relative Precision in estimating the scale parameter using RSS over SRS

\begin{tabular}{|l|lllll|}
\hline \multicolumn{1}{|c|}{$n$} & 5 & 8 & 10 & 12 & 15 \\
\hline 0.25 & 2.028766 & 2.791312 & 3.301645 & 3.813705 & 4.584696 \\
0.50 & 2.348881 & 3.353996 & 4.025434 & 4.697940 & 5.708382 \\
1.50 & 2.692024 & 3.969610 & 4.824324 & 5.680652 & 6.967306 \\
2.50 & 2.776810 & 4.126146 & 5.029660 & 5.935170 & 7.296008 \\
3.00 & 2.797785 & 4.165401 & 5.081374 & 5.999447 & 7.379242 \\
5.00 & 2.836099 & 4.238297 & 5.177844 & 6.119687 & 7.535336 \\
\hline
\end{tabular}

7.3 Estimating location and scale parameters using RSS

Let $X_{(11)}, X_{(22)}, X_{(33)}, \ldots, X_{(n n)}$ be a ranked set sample (Cycle 1) from the pdf given in (3), then $E\left\{X_{(i i)}\right\}=\mu+c_{i: n} \sigma$ and $V\left\{X_{(i i)}\right\}=d_{i: n} \sigma^{2}$, where $c_{i: n}=$ $E\left\{Y_{(i i)}\right\}, d_{i: n}=V\left\{Y_{(i i)}\right\}$. Following the method of David and Nagaraja (2003), we compute the least squares estimates $\hat{\mu}_{\text {RSSBLUE }}$ and $\hat{\sigma}_{\text {RSSBLUE }}$ of $\mu$ and $\sigma$ respectively.The relative precision of estimates are computed and shown in Table (10). 
Table 10

Relative Precision in estimating location and scale parameters using RSS over SRS

\begin{tabular}{|l|llllll|}
\hline$n$ & 5 & 6 & 7 & 8 & 10 & 15 \\
\hline$\alpha$ & \multicolumn{5}{|c|}{$V\left(\hat{\mu}_{\text {SRSBLUE }}\right) / V\left(\hat{\mu}_{\text {RSSBLUE }}\right)$} \\
\hline 0.25 & 0.93901217 & 0.969205 & 0.988601 & 1.001446 & 1.01617 & 1.029123 \\
0.50 & 0.92971395 & 0.990456 & 1.038524 & 1.077219 & 1.134978 & 1.216394 \\
2.50 & 0.9695873 & 1.094576 & 1.214898 & 1.331423 & 1.555482 & 2.079552 \\
5.00 & 0.97758532 & 1.110036 & 1.239165 & 1.365691 & 1.612841 & 2.20861 \\
\hline$\alpha$ & \multicolumn{6}{|c|}{$V\left(\hat{\sigma}_{\text {SRSBLUE }}\right) / V\left(\hat{\sigma}_{\text {RSSBLUE }}\right)$} \\
\hline 0.25 & 1.79328971 & 2.086714 & 2.371291 & 2.64963 & 3.194192 & 4.522285 \\
0.50 & 1.70705093 & 2.040913 & 2.377683 & 2.716588 & 3.398704 & 5.116085 \\
2.50 & 1.22983342 & 1.427199 & 1.622855 & 1.817114 & 2.202202 & 3.149992 \\
5.00 & 1.16515492 & 1.343524 & 1.51944 & 1.69343 & 2.036978 & 2.878687 \\
\hline
\end{tabular}

We propose the modified estimator $\hat{\sigma}^{*}$ of $\sigma$,

$$
\hat{\sigma^{*}}=\sum_{i=1}^{n} X_{(i k)} / n c_{k: n}
$$

The values of $k$ are given in Table 11 .

Table 11

The choice of $k t h$ order statistic in $i t h$ set of size $n$ (RSS

Cycle 1), when $n \in[a, b]^{1}$ for different values of $\alpha$

\begin{tabular}{|l|llllllll|}
\hline \multicolumn{1}{|c|}{$\alpha$} & 0.1 & 0.25 & 0.5 & 0.75 & 1 & 2 & 5 & 10 \\
\hline$n$ & {$[2,30]$} & {$[2,14]$} & {$[2,8]$} & {$[2,6]$} & {$[2,5]$} & {$[2,3]$} & $2^{2}$ & - \\
$n-1$ & & {$[15,27]$} & {$[9,15]$} & {$[7,11]$} & {$[6,9]$} & {$[4,6]$} & {$[3,4]$} & {$[2,3]$} \\
$n-2$ & & {$[28,30]$} & {$[16,23]$} & {$[12,17]$} & {$[10,14]$} & {$[7,10]$} & {$[5,7]$} & {$[4,5]$} \\
$n-3$ & & & {$[24,29]$} & {$[18,23]$} & {$[15,19]$} & {$[11,13]$} & {$[8,9]$} & {$[6,8]$} \\
$n-4$ & & & & {$[24,29]$} & {$[20,24]$} & {$[14,16]$} & {$[10,11]$} & {$[9,10]$} \\
$n-5$ & & & & & & {$[17,20]$} & {$[12,14]$} & {$[11,12]$} \\
$n-6$ & & & & & & {$[21,23]$} & {$[15,16]$} & {$[13,14]$} \\
$n-7$ & & & & & & {$[24,27]$} & {$[17,19]$} & {$[15,16]$} \\
$n-8$ & & & & & & & {$[20,21]$} & {$[17,18]$} \\
\hline
\end{tabular}

${ }^{1} a$ and $b$ are integers respectively.

2 Single value 
From (52),

$$
V\left(\hat{\sigma}^{*}\right)=\frac{\sigma^{2}}{n\left(c_{k: n}^{2} / d_{k: n}\right)}
$$

From (48) and (53) we have

$$
R P_{2}=\frac{V\left(\hat{\sigma}_{\mathrm{BLUESRS}}\right)}{V\left(\hat{\sigma}^{*}\right)}
$$

Table 12

Relative Precision: $\operatorname{RSS}(n=5$, Cycle 1$)$, SRS

$(n(n+1) / 2=15)$, Formula $(54)$

\begin{tabular}{llllll}
\hline$\alpha$ & 0.1 & 0.25 & 0.5 & 1.0 & 2.0 \\
RP & 1.57871 & 1.47454 & 1.34702 & 1.18739 & 1.18406 \\
\hline
\end{tabular}

7.4 Estimatiing shape parameter $\alpha$

Suppose the distribution in (1) is known for parameters $\mu$ and $\sigma$. Assume that the distribution of $Y=\frac{X-\mu}{\sigma}$ is given by

$$
\begin{gathered}
F(y ; \alpha)=\left(1-e^{-y}\right)^{1 / \alpha}, \quad \alpha, y>0 \\
f(y ; \alpha)=(1 / \alpha)\left(1-e^{-y}\right)^{(1 / \alpha)-1} e^{-y} \quad \alpha, y>0
\end{gathered}
$$

Then, distribution of $Z=-\ln F(Y)$ is

$$
F_{Z}(z)=1-e^{-z / \alpha}, \quad \alpha>0, z>0
$$

Let $Y_{(1: n)}, Y_{(2: n)}, \ldots, Y_{(n: n)}$ be an order statistics from standard exponential distribution

$$
F_{Y}(y)=1-e^{-y}, \quad y>0
$$

Then

$$
\begin{array}{r}
c_{i: n}=E\left\{Y_{(i: n)}\right\}=\sum_{j=1}^{i} \frac{1}{(n-j+1)} \\
d_{i: n}=V\left\{Y_{(i: n)}\right\}=\sum_{j=1}^{i} \frac{1}{(n-j+1)^{2}} \\
\operatorname{Cov}\left(Y_{(r: n)}, Y_{(s: n)}\right)=V\left\{Y_{(r: n)}\right\}, \quad r<s
\end{array}
$$

Let $Z_{(11)}, Z_{(22)}, \ldots, Z_{(n n)}$ be an RSS from the distribution given in (57), then we write an estimator of $\alpha$ based on RSS as

$$
\hat{\alpha}_{\mathrm{RSS}-\mathrm{BLUE}}=\sum_{i=1}^{n} w_{i} Z_{(i i)}
$$


Where,

$$
w_{i}=\left(c_{i: n} / d_{i: n}\right) / \sum_{i=1}^{n}\left(c_{i: n}^{2} / d_{i: n}\right)
$$

and

$$
V\left(\hat{\alpha}_{\mathrm{RSS}-\mathrm{BLUE}}\right)=\sigma^{2} / \sum_{i=1}^{n}\left(c_{i: n}^{2} / d_{i: n}\right)
$$

The Table 13 shows the relative precision of estimate $\hat{\alpha}_{\mathrm{RSS}}$

Table 13

Relative Precision: $V\left(\hat{\alpha}_{\mathrm{SRS}}\right) / V\left(\hat{\alpha}_{\mathrm{RSS}}\right)$

\begin{tabular}{lllllll}
\hline$n$ & 5 & 6 & 7 & 8 & 9 & 10 \\
$R P$ & 2.59255 & 2.9908 & 3.38957 & 3.7888 & 4.18844 & 4.58844 \\
\hline
\end{tabular}

Also, we suggest the modified ranked set sampling scheme to obtain the relative precision of estimate of $\alpha$ using ordered least squares. The proposed estimate of $\alpha$ and the variance of estimate respectively are

$$
\begin{array}{r}
\hat{\alpha}^{*}=\sum_{i=1}^{n} Z_{(i k)} / n c_{k: n} \\
V\left(\hat{\alpha^{*}}\right)=\frac{\sigma^{2}}{n\left(c_{k: n}^{2} / d_{k: n}\right)}
\end{array}
$$

Where the value of $k$ is

$$
k= \begin{cases}n & 2 \leq n \leq 3 \\ n-1 & 4 \leq n \leq 6 \\ n-2 & 7 \leq n \leq 10 \\ n-3 & 11 \leq n \leq 13 \\ n-4 & 14 \leq n \leq 16 \\ n-5 & 17 \leq n \leq 20\end{cases}
$$

Table 14

Relative Precision: $V\left(\hat{\alpha}_{\text {SRS-BLUE }}\right) / V\left(\hat{\alpha}^{*}\right)$

\begin{tabular}{lrrrrr}
\hline$n$ & 5 & 10 & 12 & 15 & 20 \\
$\mathrm{RP}$ & 3.562156 & 6.81178 & 8.16024 & 10.0530 & 13.2911 \\
\hline
\end{tabular}




\section{Simulated Data}

Based on the computer program developed by the author, we generate RSS sample of size $n=10$ from the density function given in (2) for known values of $\alpha=0.5,2.0$, and $\mu=10, \sigma=5$ respectively. We refer to the Table 20 (Page 35 ) of coefficients to estimate $\mu$ and $\sigma$.

\section{Table 15}

Ranked Set Sample of size $n=10$ to estimate the parameters $\mu$ and $\sigma$ of probability density given in (2)

\begin{tabular}{l|lllll|ll}
\hline \multirow{2}{*}{$\alpha=2.0$} & 1 & 2 & 3 & 4 & 5 & $\hat{\mu}=$ & 10.17431 \\
\cline { 2 - 8 } & 11.04929 & 13.35352 & 13.85929 & 16.04259 & 18.13354 & & \\
\hline \multirow{2}{*}{$\alpha=0.5$} & 6 & 8 & 9 & 10 & $\hat{\sigma}=$ & 4.951395 \\
& 12.5353 & 23.59186 & 18.56782 & 15.65142 & 33.38607 & & \\
\cline { 2 - 8 } & 1 & 2 & 3 & 4 & 5 & $\hat{\mu}=$ & 10.1829 \\
& 10.32787 & 10.1165 & 11.31832 & 10.80349 & 10.61852 & & \\
\hline
\end{tabular}

\section{Simulation Study}

The estimates $(\hat{\alpha}, \hat{\beta})$ are determined numerically, the steps are

1. Generate 5000 random numbers from density function given in (4) with $\alpha=0.5,4.0$ and $\beta=0.5$

2. In the first cycle select Moving Extreme Ranked Set Sample from $m(m+1)$ observations given in step (1). Where $m=7$

3. In the first cycle select Quartile ranked set sample and Median ranked set sample from $m^{2}$ observations each given in step (1).

4. In the first cycle select Simple Random Sample of size $m$.

5. Repeat the steps (1) to (5) for $h=2,3,4,5$ cycles. The sample size of MERSS is $2 \mathrm{hm}$, the sample size of QRSS, MRSS and FLERSS respectively is $2 \mathrm{hm}$. The sample size of SRS is $2 \mathrm{hm}$.

6. Using $\mathrm{R} \operatorname{nlm}($ ) function and $\mathrm{R}$ maxLik package find the estimates $(\hat{\alpha}, \hat{\beta})$, the elements of observed Fisher Information matrix and 95\% confidence limits of the parameters $\alpha$ and $\beta$ numerically using 500 simulation runs.

7. From the numerical values obtained in step (6) for 500 simulation run determine average values of mse, bias, confidence limits, entries of observed Fisher information matrix respectively. Compute the observed variance- covariance matrix from the observed Fisher information matrix.The efficiency and asymptotic efficiency of the estimates respectively are given in Tables (4) to (8) and and Tables (16) to (18). 


\section{Conclusion}

1. We obtain numerically the estimates of parameters of generalized exponential distribution given in (1) and investigate their performances, using ranked set sampling scheme and simple random sampling scheme with the method of least squares

(a) When shape parameter $\alpha$ is unknown, the estimator of shape parameter using RSS is efficient than that of using SRS and (b) When shape parameter $\alpha$ is known and is more than 1, the estimator of location parameter using RSS is efficient than that of using SRS for sample size 6 or more. For any value of shape parameter $\alpha>0$, the estimator of scale parameter is efficient than that of using Simple Random Sampling for sample size $n \geq 5$. (The numerical comutations are given in Tables (9) and (10))

2. We use real data (Table 1) to compare the performance of SRS and the MERSS scheme. MERSS is asymptotically more efficient than SRS.

3. In this paper, we examine different modifications of ranked set sampling and compare the estimated parameter values of the generalized exponential distribution given in (3). The MERSS scheme is asymptotically more efficient than other schemes such as SRS, QRSS, MRSS, and FLERSS for cycles $h=2,3,4,5$ to estimate both shape and scale parameters of distribution given in (3). The numerical computations are supplied in Tables (4) to (8) and Tables (16) to (18). respectively. 
Table 16

Estimate of shape and scale parameters $(\alpha=4.0, \beta=0.5)$ for different cycles $h$

\begin{tabular}{rlllllll}
\hline Sampling & $h$ & $\hat{\alpha}$ & $\hat{\beta}$ & $\operatorname{bias}(\hat{\alpha})$ & $\operatorname{bias}(\hat{\beta})$ & $\operatorname{MSE}(\hat{\alpha})$ & $\operatorname{MSE}(\hat{\beta})$ \\
\hline SRS & 2 & 4.49995 & 0.52013 & 0.49995 & 0.02013 & 3.66020 & 0.01182 \\
MERSS & 2 & 4.19172 & 0.50895 & 0.19172 & 0.00895 & 1.36022 & 0.00520 \\
QRSS & 2 & 4.48234 & 0.51387 & 0.48234 & 0.01387 & 3.89591 & 0.00867 \\
MRSS & 2 & 4.44518 & 0.51261 & 0.44518 & 0.01261 & 2.90570 & 0.00750 \\
FLERSS & 2 & 4.27402 & 0.50665 & 0.27402 & 0.00665 & 1.88851 & 0.00589 \\
\hline SRS & 3 & 4.25081 & 0.50958 & 0.25081 & 0.00958 & 1.76369 & 0.00619 \\
MERSS & 3 & 4.07897 & 0.50235 & 0.07897 & 0.00235 & 0.68847 & 0.00317 \\
QRSS & 3 & 4.24073 & 0.50557 & 0.24073 & 0.00557 & 1.63238 & 0.00545 \\
MRSS & 3 & 4.17018 & 0.50206 & 0.17018 & 0.00206 & 1.73647 & 0.00535 \\
FLERSS & 3 & 4.09946 & 0.50084 & 0.09946 & 0.00084 & 1.03079 & 0.00354 \\
\hline SRS & 4 & 4.13877 & 0.50309 & 0.13877 & 0.00309 & 1.11079 & 0.00441 \\
MERSS & 4 & 3.95781 & 0.50054 & -0.04219 & 0.00054 & 0.50547 & 0.00248 \\
QRSS & 4 & 4.17945 & 0.50469 & 0.17945 & 0.00469 & 1.38519 & 0.00483 \\
MRSS & 4 & 4.04921 & 0.49722 & 0.04921 & -0.00278 & 1.09618 & 0.00395 \\
FLERSS & 4 & 4.08016 & 0.49969 & 0.08016 & -0.00031 & 0.85204 & 0.00279 \\
\hline SRS & 5 & 4.027300 & 0.499034 & 0.027300 & -0.000966 & 0.803603 & 0.003417 \\
MERSS & 5 & 3.972811 & 0.498362 & -0.027189 & -0.001638 & 0.395034 & 0.002063 \\
QRSS & 5 & 4.098269 & 0.499719 & 0.098269 & -0.000281 & 0.945214 & 0.003286 \\
MRSS & 5 & 3.991335 & 0.493423 & -0.008665 & -0.006577 & 0.827605 & 0.003141 \\
FLERSS & 5 & 3.969677 & 0.495252 & -0.030323 & -0.004748 & 0.544031 & 0.002092 \\
\hline
\end{tabular}




\section{Table 17}

$\alpha=4.0, \beta=0.5$ :

Observed Fisher Information (FI),Variance- Covariance(VC) and 95\% Confidence limits for different cycles $h$.

\begin{tabular}{|c|c|c|c|c|c|c|c|c|c|}
\hline \multirow[t]{2}{*}{ Sampling } & \multirow{2}{*}{$\begin{array}{c}h \\
2\end{array}$} & \multirow{2}{*}{$\begin{array}{l}\text { Est } \\
\hat{\alpha}\end{array}$} & \multicolumn{2}{|c|}{ FI } & \multicolumn{2}{|c|}{$\mathrm{VC}$} & \multicolumn{2}{|c|}{$95 \% \mathrm{CI}(\alpha, \beta)$} & \multirow{2}{*}{$\begin{array}{c}\text { Width } \\
6.12019\end{array}$} \\
\hline & & & 2.02741 & -20.57385 & 1.20213 & 0.06986 & 1.43985 & 7.56004 & \\
\hline \multirow{7}{*}{ SRS } & & $\hat{\beta}$ & -20.57385 & 354.04789 & 0.06986 & 0.00688 & 0.33086 & 0.70939 & 0.37853 \\
\hline & 3 & $\hat{\alpha}$ & 2.91944 & -30.81175 & 0.89139 & 0.05200 & 1.94695 & 6.55466 & 4.60771 \\
\hline & & $\hat{\beta}$ & -30.81175 & 528.12938 & 0.05200 & 0.00493 & 0.35771 & 0.66145 & 0.30374 \\
\hline & 4 & $\hat{\alpha}$ & 3.85482 & -41.43064 & 0.69793 & 0.04080 & 2.21751 & 6.06002 & 3.84251 \\
\hline & & $\hat{\beta}$ & -41.43064 & 708.70738 & 0.04080 & 0.00380 & 0.37302 & 0.63315 & 0.26013 \\
\hline & 5 & $\hat{\alpha}$ & 4.92411 & -52.37682 & 0.55172 & 0.03278 & 2.37133 & 5.68327 & 3.31194 \\
\hline & & $\hat{\beta}$ & -52.37682 & 881.64748 & 0.03278 & 0.00308 & 0.38345 & 0.61462 & 0.23117 \\
\hline \multirow{8}{*}{ QRSS } & 2 & $\hat{\alpha}$ & 7.02543 & -85.96232 & 0.57291 & 0.03519 & 2.20059 & 6.76408 & 4.56349 \\
\hline & & $\hat{\beta}$ & -85.96232 & 1399.54567 & 0.03519 & 0.00288 & 0.38238 & 0.64535 & 0.26298 \\
\hline & 3 & $\hat{\alpha}$ & 10.38784 & -130.45630 & 0.42877 & 0.02648 & 2.52354 & 5.95793 & 3.43439 \\
\hline & & $\hat{\beta}$ & -130.45633 & 2112.67340 & 0.02648 & 0.00211 & 0.39953 & 0.61161 & 0.21208 \\
\hline & 4 & $\hat{\alpha}$ & 13.90917 & -174.48610 & 0.32989 & 0.02057 & 2.71919 & 5.63972 & 2.92053 \\
\hline & & $\hat{\beta}$ & -174.48610 & 2798.84540 & 0.02057 & 0.00164 & 0.41280 & 0.59658 & 0.18378 \\
\hline & 5 & $\hat{\alpha}$ & 17.03452 & -218.18920 & 0.28552 & 0.01771 & 2.82825 & 5.36829 & 2.54005 \\
\hline & & $\hat{\beta}$ & -218.18915 & 3518.02810 & 0.01771 & 0.00138 & 0.41824 & 0.58120 & 0.16296 \\
\hline \multirow{8}{*}{ MRSS } & 2 & $\hat{\alpha}$ & 7.48840 & -101.35460 & 0.81440 & 0.05030 & 1.43133 & 7.45903 & 6.02770 \\
\hline & & $\hat{\beta}$ & -101.35460 & 1640.88370 & 0.05030 & 0.00372 & 0.34355 & 0.68168 & 0.33813 \\
\hline & 3 & $\hat{\alpha}$ & 11.68423 & -156.86330 & 0.59861 & 0.03821 & 1.91439 & 6.42597 & 4.51158 \\
\hline & & $\hat{\beta}$ & -156.86329 & 2457.24690 & 0.03821 & 0.00285 & 0.36608 & 0.63804 & 0.27196 \\
\hline & 4 & $\hat{\alpha}$ & 15.54629 & -210.43350 & 0.48951 & 0.03141 & 2.17294 & 5.92547 & 3.75252 \\
\hline & & $\hat{\beta}$ & -210.43351 & 3279.33360 & 0.03141 & 0.00232 & 0.38018 & 0.61426 & 0.23407 \\
\hline & 5 & $\hat{\alpha}$ & 19.31565 & -264.33100 & 0.42115 & 0.02699 & 2.34902 & 5.63365 & 3.28464 \\
\hline & & $\hat{\beta}$ & -264.33097 & 4124.32300 & 0.02699 & 0.00197 & 0.38955 & 0.59730 & 0.20775 \\
\hline \multirow{8}{*}{ FLERSS } & 2 & $\hat{\alpha}$ & 7.27041 & -94.72299 & 0.72635 & 0.04519 & 1.86360 & 6.68444 & 4.82083 \\
\hline & & $\hat{\beta}$ & -94.72299 & 1522.38802 & 0.04519 & 0.00347 & 0.36242 & 0.65088 & 0.28847 \\
\hline & 3 & $\hat{\alpha}$ & 10.70515 & -142.76810 & 0.57097 & 0.03581 & 2.23884 & 5.96009 & 3.72125 \\
\hline & & $\hat{\beta}$ & -142.76812 & 2276.45010 & 0.03581 & 0.00269 & 0.38372 & 0.61796 & 0.23423 \\
\hline & 4 & $\hat{\alpha}$ & 15.54629 & -210.43350 & 0.48951 & 0.03141 & 2.17294 & 5.92547 & 3.75252 \\
\hline & & $\hat{\beta}$ & -210.43351 & 3279.33360 & 0.03141 & 0.00232 & 0.38018 & 0.61426 & 0.23407 \\
\hline & 5 & $\hat{\alpha}$ & 17.93984 & -240.66080 & 0.37109 & 0.02351 & 2.59104 & 5.34831 & 2.75727 \\
\hline & & $\hat{\beta}$ & -240.66081 & 3799.11100 & 0.02351 & 0.00175 & 0.40528 & 0.58522 & 0.17994 \\
\hline \multirow{8}{*}{ MERSS } & 2 & $\hat{\alpha}$ & 14.22281 & -148.76610 & 0.20742 & 0.01311 & 3.11274 & 5.27070 & 2.15796 \\
\hline & & $\hat{\beta}$ & -148.76607 & 2354.00030 & 0.01311 & 0.00125 & 0.43279 & 0.58511 & 0.15232 \\
\hline & 3 & $\hat{\alpha}$ & 21.02721 & -225.12810 & 0.14748 & 0.00933 & 3.23159 & 4.92635 & 1.69476 \\
\hline & & $\hat{\beta}$ & -225.12807 & 3557.46340 & 0.00933 & 0.00087 & 0.44090 & 0.56380 & 0.12290 \\
\hline & 4 & $\hat{\alpha}$ & 29.04885 & -304.14470 & 0.10863 & 0.00709 & 3.25053 & 4.66510 & 1.41457 \\
\hline & & $\hat{\beta}$ & -304.14467 & 4661.78460 & 0.00709 & 0.00068 & 0.44716 & 0.55392 & 0.10676 \\
\hline & 5 & $\hat{\alpha}$ & 35.46128 & -379.29750 & 0.09042 & 0.00582 & 3.33762 & 4.60800 & 1.27039 \\
\hline & & $\hat{\beta}$ & -379.29746 & 5895.81370 & 0.00582 & 0.00054 & 0.45087 & 0.54586 & 0.09499 \\
\hline
\end{tabular}


Table 18

Efficiency and Asymptotic Efficiency of MERSS when $\alpha=4.0, \beta=0.5$ for different cycles $h$.

\begin{tabular}{llllll} 
& & \multicolumn{2}{c}{ Efficiency } & \multicolumn{2}{l}{ Asymptotic Efficiency } \\
\cline { 3 - 6 } Sampling & $h$ & $\hat{\alpha}$ & $\hat{\beta}$ & $\hat{\alpha}$ & $\hat{\beta}$ \\
\hline \multirow{4}{*}{ SRS } & 2 & 1.073533 & 1.021956 & 5.795746 & 5.493002 \\
& 3 & 1.042127 & 1.014394 & 6.043929 & 5.652466 \\
& 4 & 1.045721 & 1.005090 & 6.425024 & 5.608347 \\
& 5 & 1.013716 & 1.001348 & 6.101925 & 5.666158 \\
\hline \multirow{4}{*}{ QRSS } & 2 & 1.069332 & 1.009651 & 2.762103 & 2.294811 \\
& 3 & 1.039657 & 1.006412 & 2.907232 & 2.418418 \\
& 4 & 1.056001 & 1.008303 & 3.036885 & 2.422000 \\
& 5 & 1.031579 & 1.002723 & 3.157809 & 2.542178 \\
\hline \multirow{4}{*}{ MRSS } & 2 & 1.060468 & 1.007190 & 3.926413 & 2.965705 \\
& 3 & 1.022360 & 0.999426 & 4.058762 & 3.265155 \\
& 4 & 1.023092 & 0.993373 & 4.506344 & 3.428378 \\
& 5 & 1.004663 & 0.990090 & 4.657791 & 3.626820 \\
\hline \multirow{4}{*}{ FLERSS } & 2 & 1.019635 & 0.995476 & 3.501908 & 2.767956 \\
& 3 & 1.005023 & 0.996997 & 3.871371 & 3.080053 \\
& 4 & 1.030912 & 0.998312 & 4.144899 & 3.077274 \\
& 5 & 0.999211 & 0.993760 & 4.104131 & 3.222161 \\
\hline
\end{tabular}




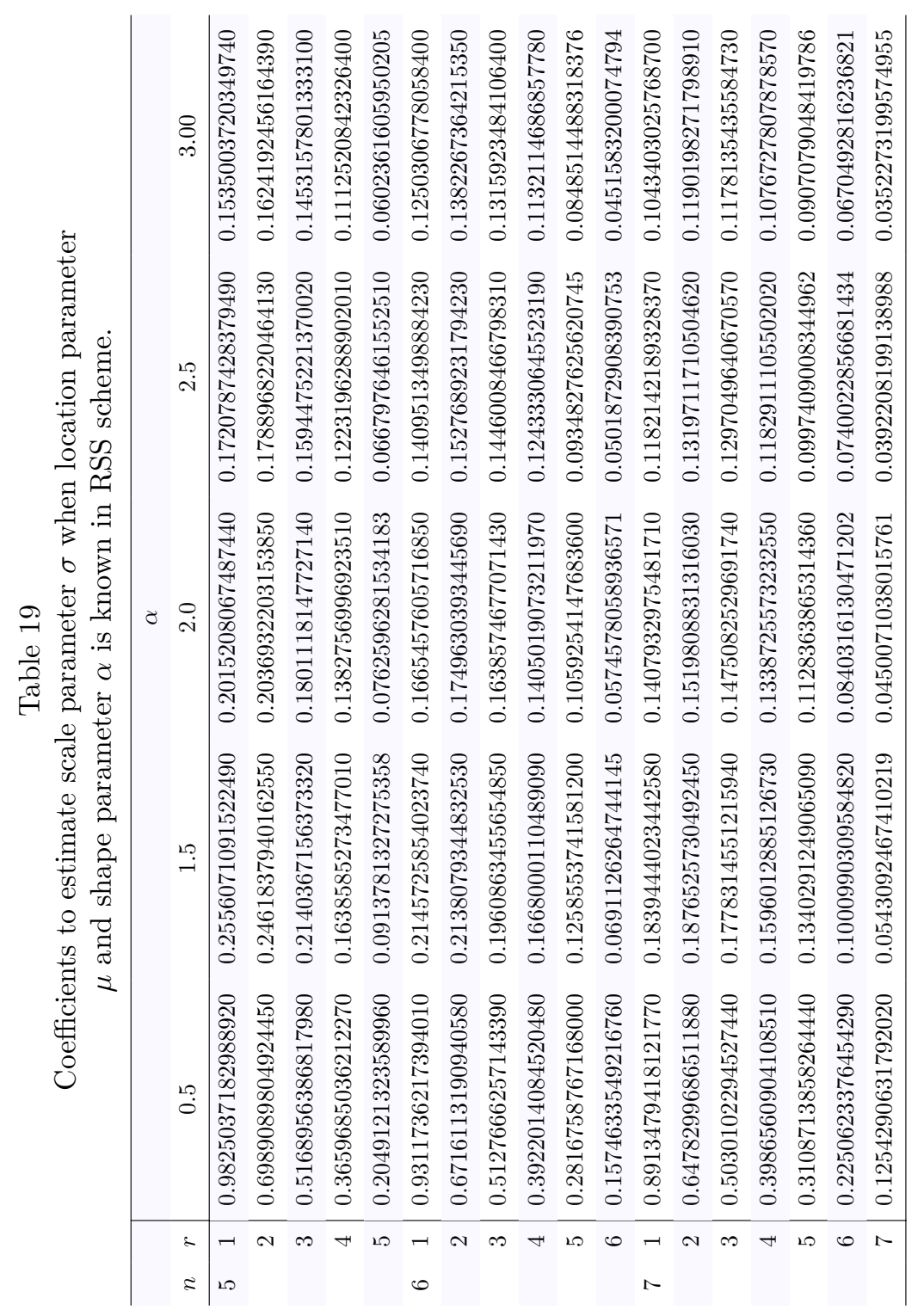




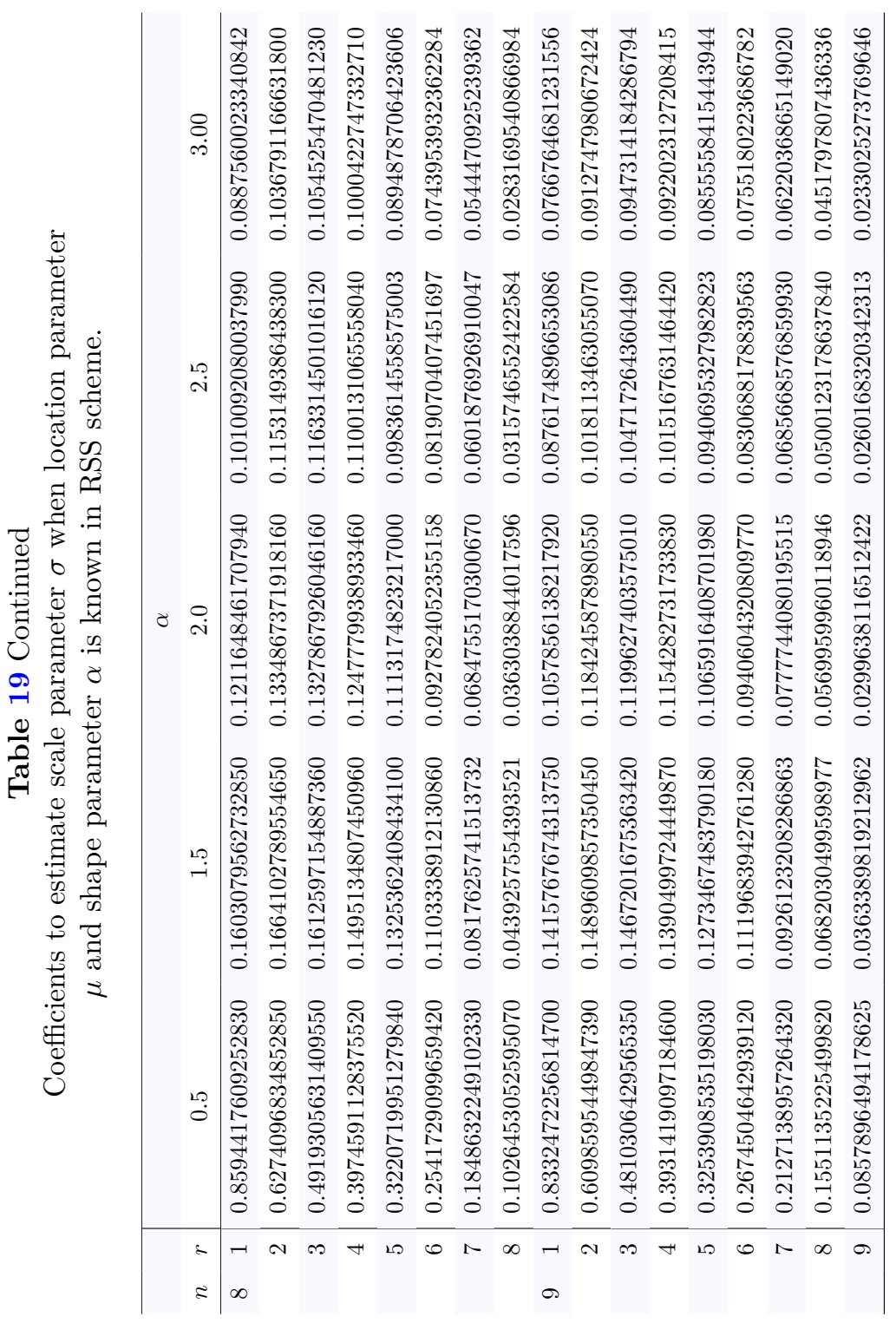




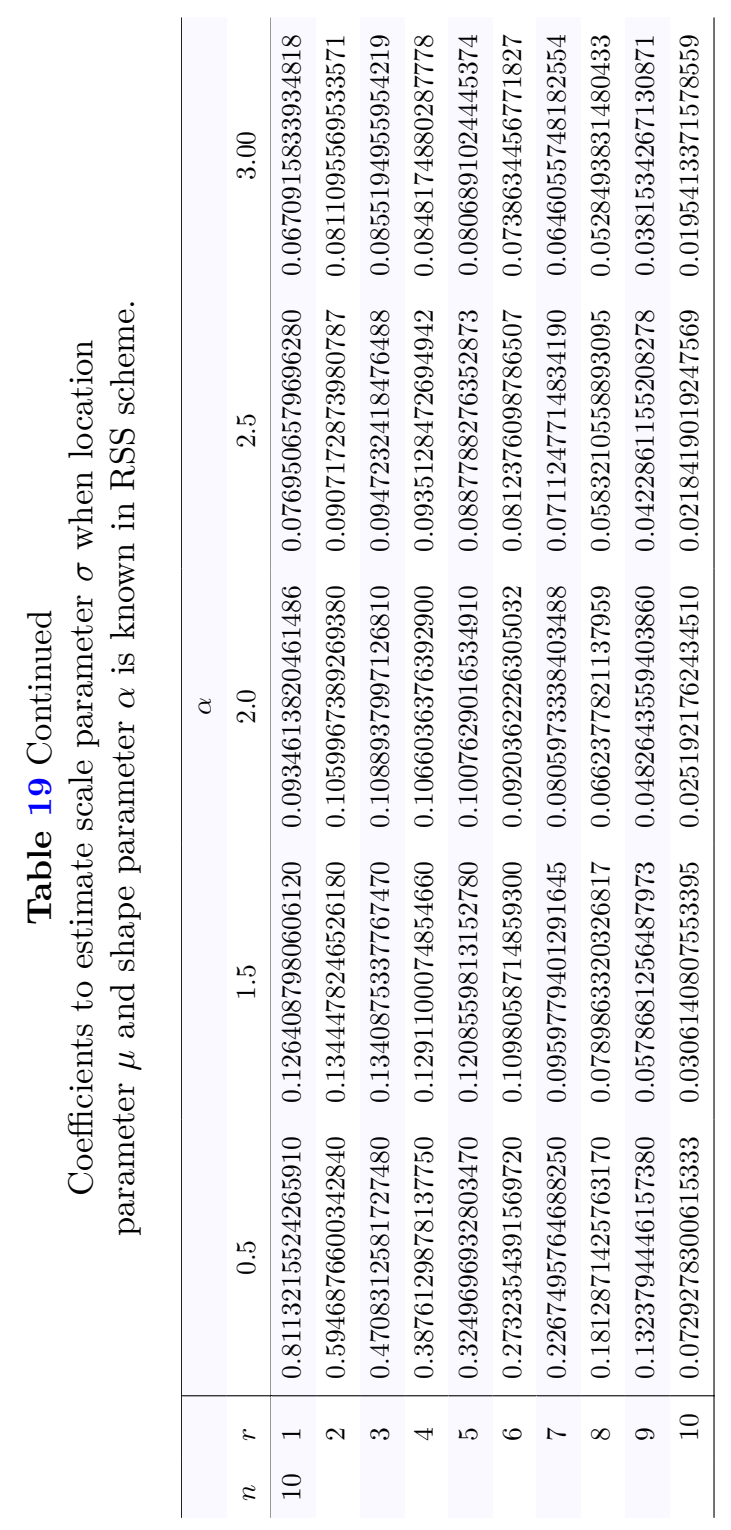




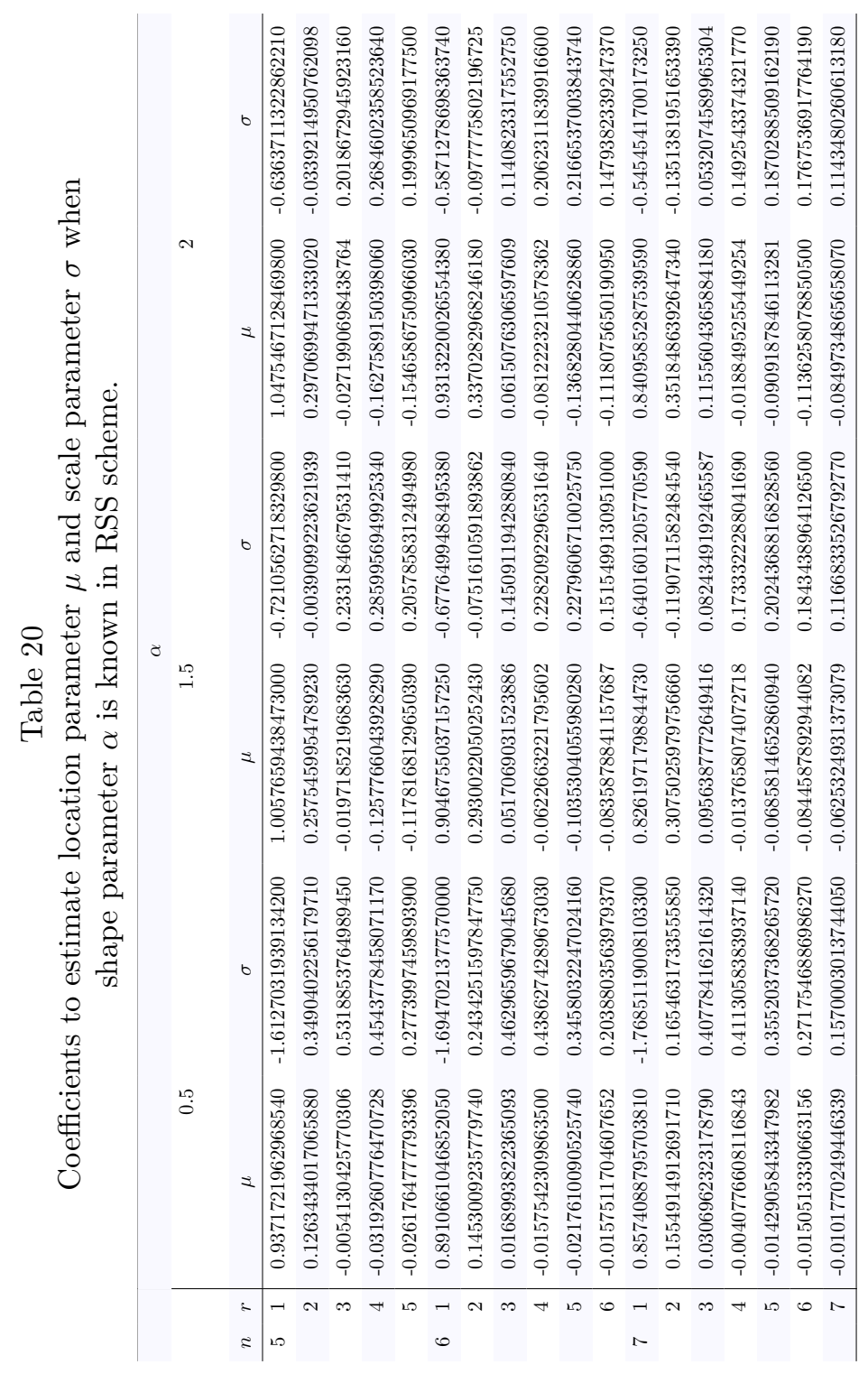




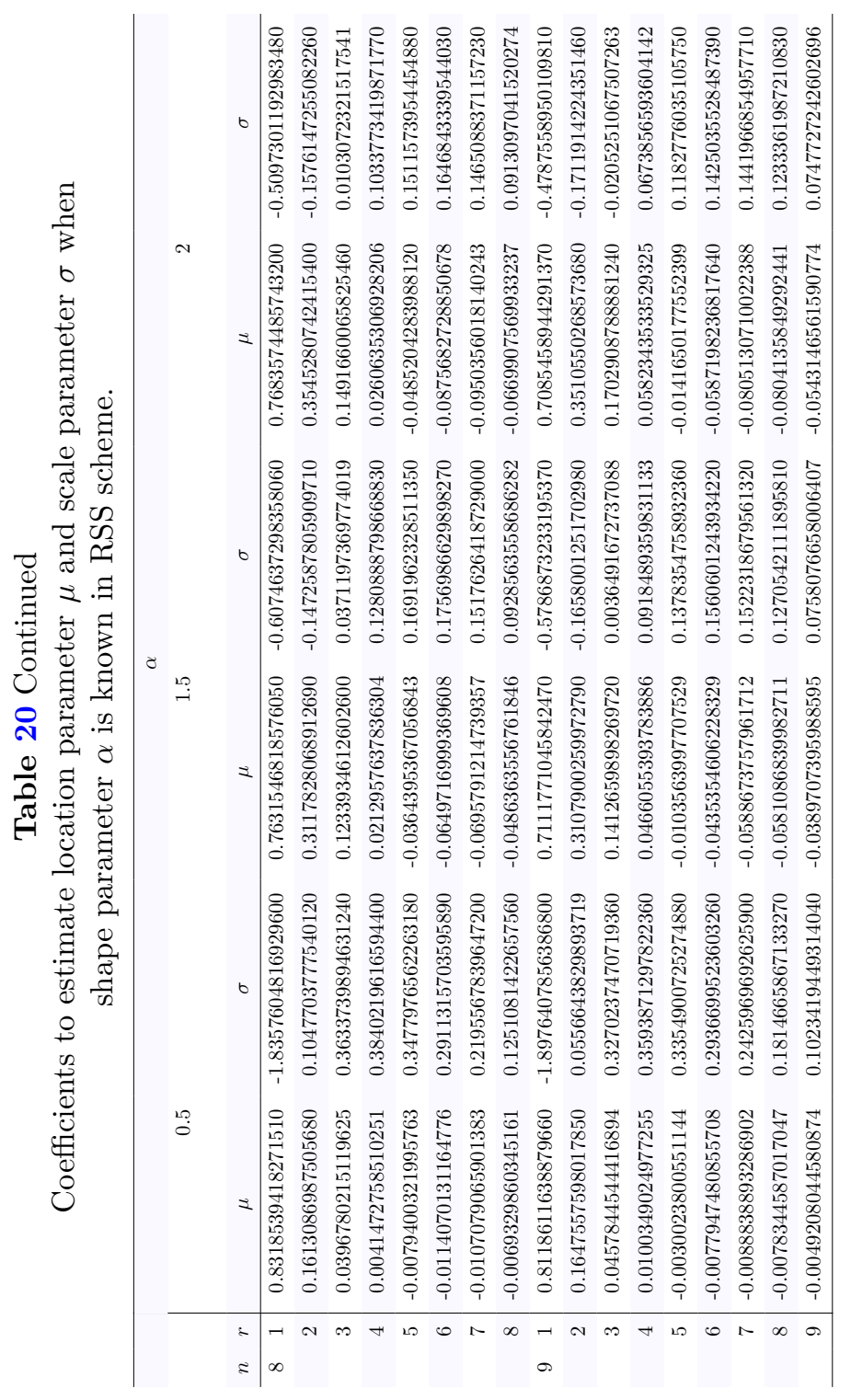




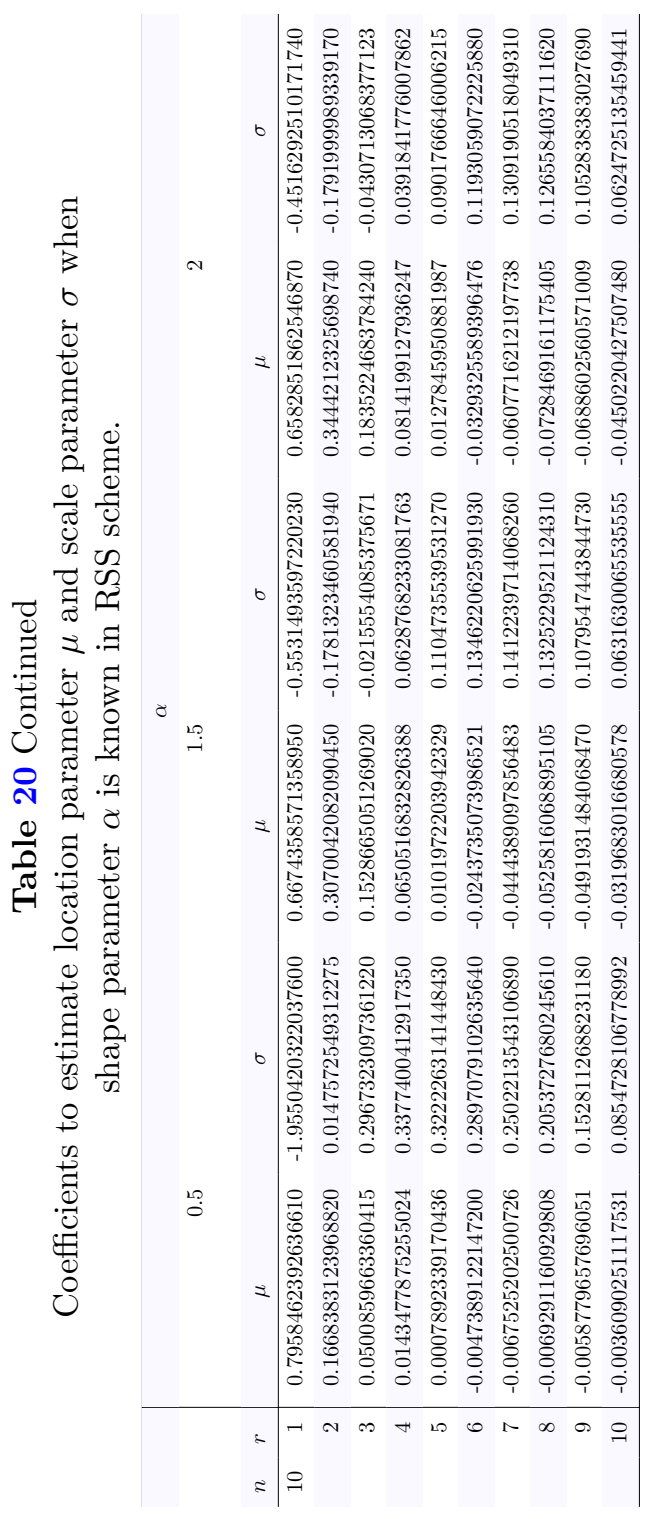




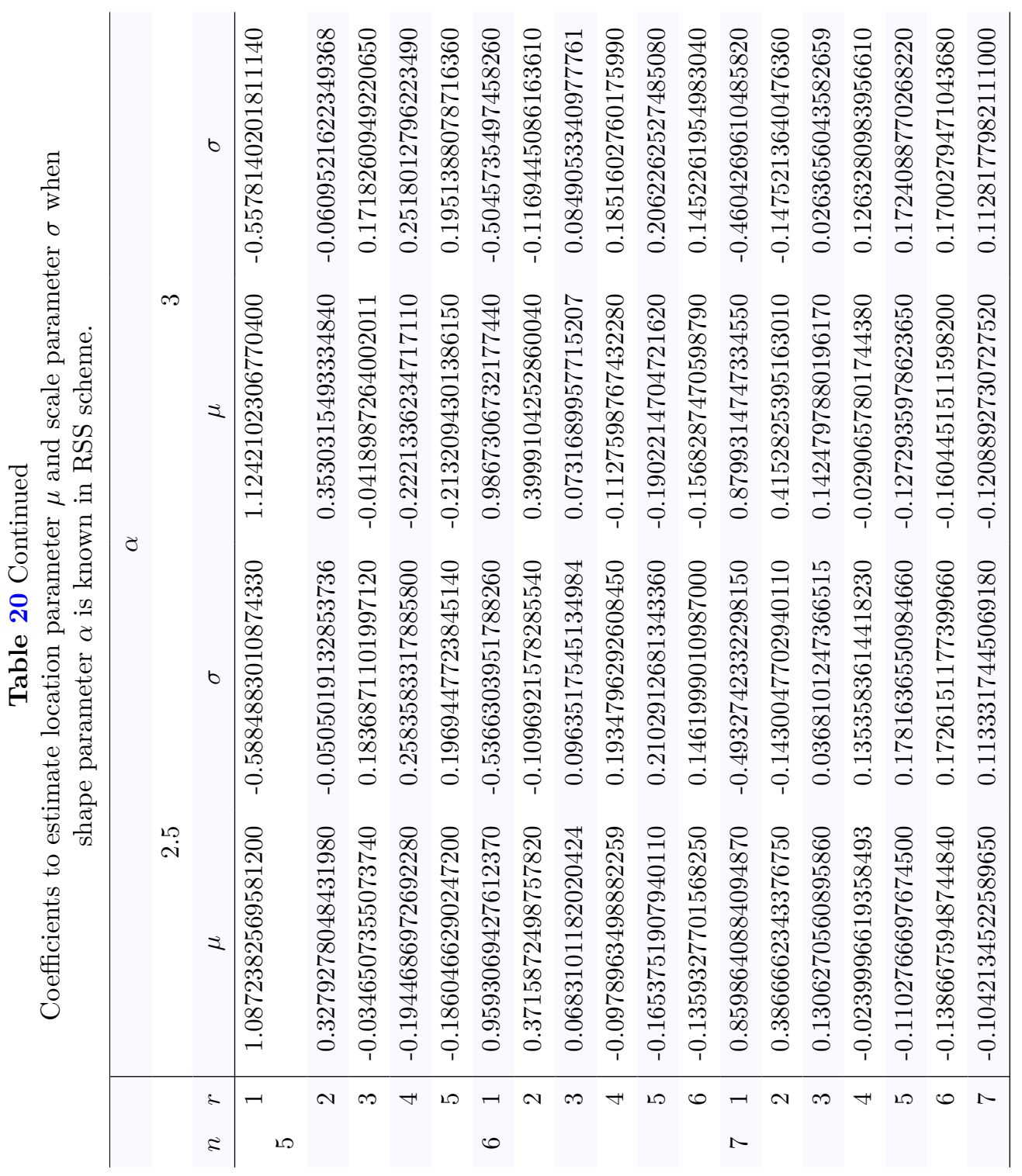




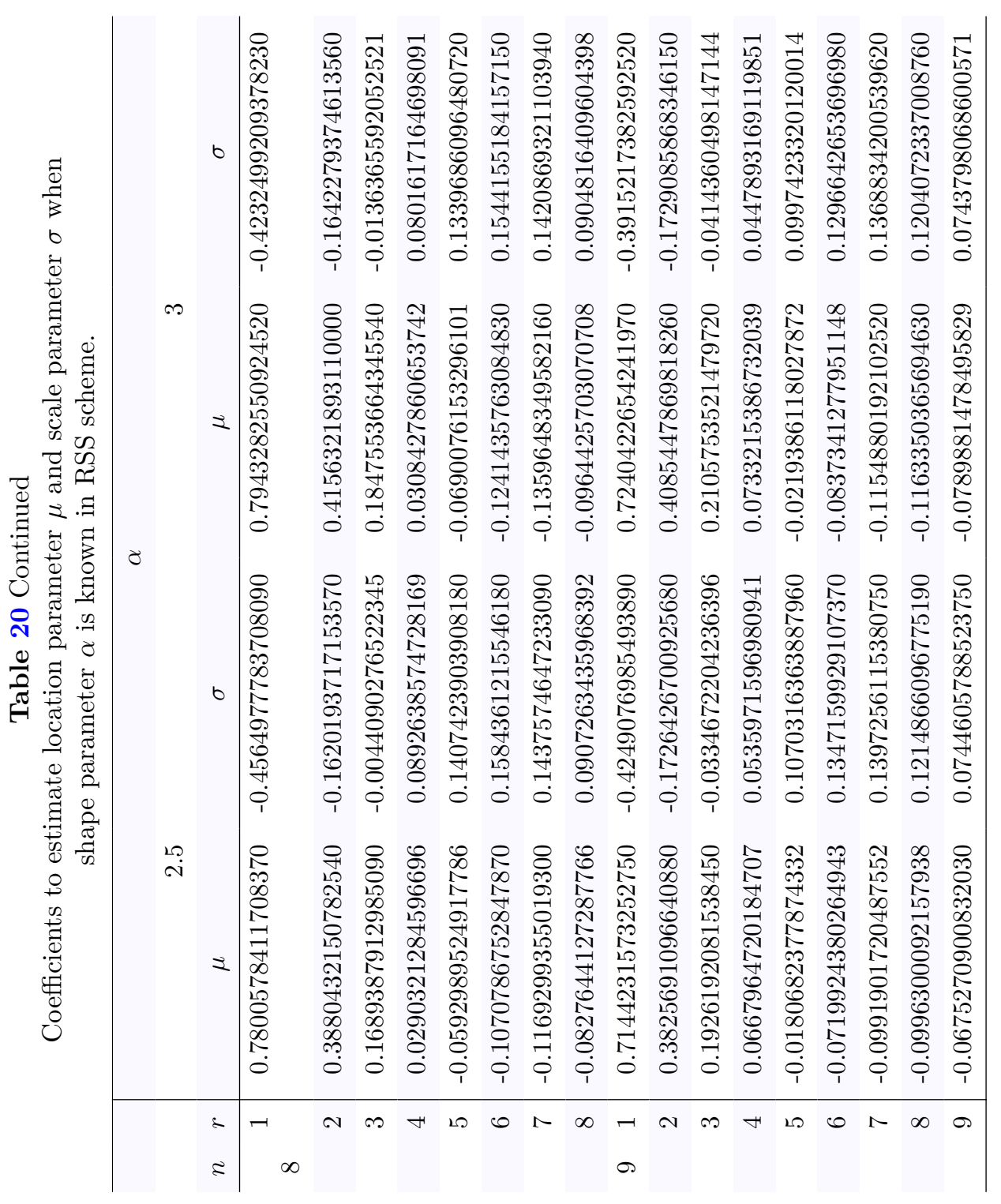




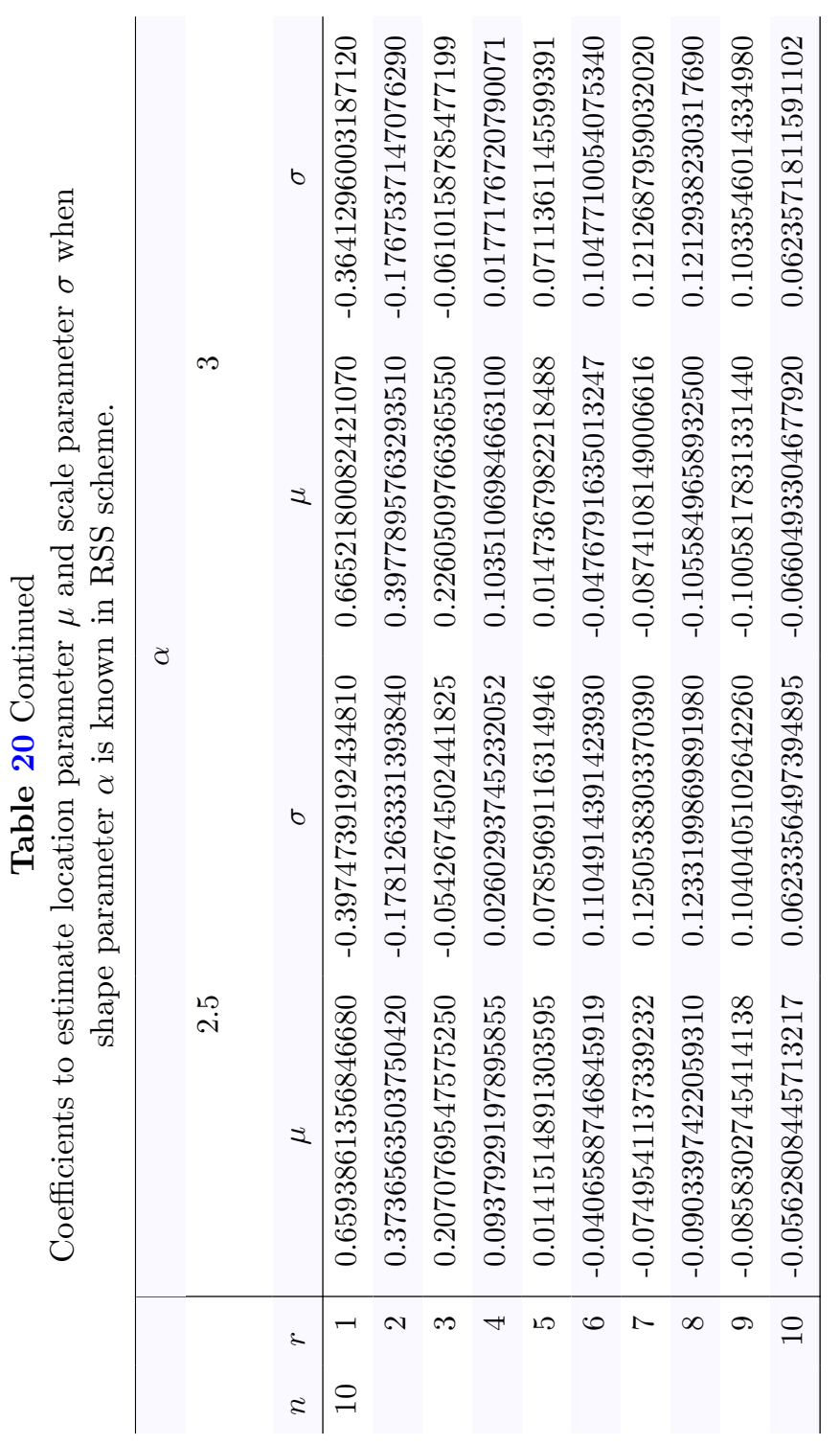




\section{References}

Al-Odat MT, Al-Saleh MF (2001) A variation of ranked set sampling. Journal of Applied Statistical Science 10(2):137-146

Chen D, Lio Y (2010) Parameter estimations for generalized exponential distribution under progressive type-i interval censoring. Computational Statistics \& Data Analysis 54(6):1581-1591

David HA, Nagaraja HN (2003) Order statistics, 3rd edn. Wiley Series in Probability and Statistics, John Wiley

Eftekharian A, Razmkhah M (2016) A unified ranked set sampling for estimating the population mean. Istatistik Journal of The Turkish Statistical Association 9(3):107-118

Eftekharian A, Razmkhah M, Ahmadi J (2021) A flexible ranked set sampling schemes: Statistical analysis on scale parameter. Statistics, Optimization \& Information Computing 9(1):189-203

Gupta RD, Kundu D (1999) Generalized exponential distributions. Australian \& New Zealand Journal of Statistics 41, DOI 10.1111/1467-842x.00072

Gupta RD, Kundu D (2001) Exponentiated exponential family: an alternative to gamma and weibull distributions. Biometrical Journal: Journal of Mathematical Methods in Biosciences 43(1):117-130

Gupta RD, Kundu D (2007) Generalized exponential distribution: existing results and some recent developments. Journal of Statistical Planning and Inference 137, DOI 10.1016/j.jspi.2007.03.030

Hajebi M, Rezaei S, Nadarajah S (2012) Confidence intervals for $P(Y<X)$ for the generalized exponential distribution. Statistical Methodology 9(3):445-455

Henningsen A, Toomet $\mathrm{O}$ (2011) maxlik: A package for maximum likelihood estimation in $\mathrm{r}$. Computational Statistics 26(3):443-458

Khan DMSH (1987) A generalized exponential distribution. Biometrical Journal 29:121-127, DOI 10.1002/bimj.4710290121

Kundu D, Gupta RD (2005) Estimation of $\mathrm{P}[Y<X]$ for generalized exponential distribution. Metrika 61(3):291-308

Kundu D, Gupta RD (2011) Absolute continuous bivariate generalized exponential distribution. AStA Advances in Statistical Analysis 95, DOI 10.1007/s10182-010-0151-0

Lieblein J, Zelen M (1956) Statistical investigation of the fatigue life of deep-groove ball bearings. Journal of research of the national bureau of standards 57(5):273-316

McIntyre GA (1952) A method for unbiased selective sampling, using ranked sets. Australian Journal of Agricultural Research 3

Muttlak HA (1997) Median ranked set sampling. J Appl Stat Sci 6:245-255

Muttlak HA (2003) Investigating the use of quartile ranked set samples for estimating the population mean. Applied Mathematics and Computation 146(2-3):437-443

Patil GP, Sinha AK, Taillie C (1995) Finite population corrections for ranked set sampling. Annals of the Institute of Statistical Mathematics 47:621-636, DOI 10.1007/bf01856537

Raqab, Madi MZ, T M, Debasis K (2008) Estimation of $p(y<x)$ for the three parameter generalized exponential distribution. Communication in Statistics- Theory and Methods 37:2854-2864, DOI 10.1080/03610920802162664

Raqab Mohammad M, Ahsanullah M (2001) Estimation of the location and scale parameters of generalized exponential distribution based on order statistics. Journal of statistical Computation and simulation 69(2):109-123

Samuh MH, Qtait A (2015) Estimation for the parameters of the exponentiated exponential distribution using a median ranked set sampling. Journal of Modern Applied Statistical Methods 14(1):19

Tahmasebi S, Jafari AA (2014) Estimators for the parameter mean of morgenstern type bivariate generalized exponential distribution using ranked set sampling. arXiv preprint arXiv:14052680

Zehua Chen, Zhidong Bai, Bimal K Sinha (2004) Ranked set sampling: Theory and Applications, vol 176. Springer Science \& Business Media 